\title{
Strain-Release Electrophilic Activation via E-Cycloalkenones
}

\author{
Joseph Moran, Peter Dornan, and André M. Beauchemin* \\ Department of Chemistry, University of Ottawa, 10 Marie Curie, Ottawa, Ontario, Canada, K1N 6N5 \\ andre.beauchemin@uottawa.ca
}

\section{Supporting Information}

\section{Table of Contents}

\section{General Information}

S1

Materials

$\mathrm{S} 2$

General Procedure for Solvent Scan (Table 1)

$\mathrm{S} 2$

General Procedure for Photoinduced 1,4-Addition of Nitrogen Heterocycles (Table 2 and 3 )

Procedure for Deuterium Labelling Experiment for Determination of Stereochemistry

$\mathrm{S} 10$

Procedure for Low Temperature Generation/Trapping of $(E)$-Cyclooct-2-enone

S10

Procedure for Low Temperature NMR experiments with $(E)$-Cyclooct-2-enone

$\mathrm{S} 11$

${ }^{1} \mathrm{H}$ and ${ }^{13} \mathrm{C}$ NMR spectra for all new compounds

$\mathrm{S} 12$

General Information. All reactions were performed in air-dried or oven-dried borosilicate tubes. Reactions were either carried out in a Rayonet photoreactor or between two Luzchem exposure panels equipped with four ultraviolet lamps each. UV-A bulbs $(\sim 350 \mathrm{~nm})^{1,2}$ and exposure panels were purchased from Luzchem Research, Inc. Purification of reaction products was carried out by flash column chromatography using silica gel $(40-63 \mu \mathrm{m})$. Analytical thin layer chromatography was performed on aluminum sheets pre-coated with silica gel 60 F254, cut to size. Visualization was accomplished with UV light and an aqueous vanillin solution followed by heating.

Infrared spectra were recorded on a Bruker Equinox 55 spectrometer. ${ }^{1} \mathrm{H}$ NMR spectra were recorded on a Bruker Avance300 (300 MHz) or Avance400 (400 MHz) spectrometer at ambient temperature and are reported in ppm using solvent as the internal standard $\left(\mathrm{CDCl}_{3}\right.$ at $7.26 \mathrm{ppm}$ or $\mathrm{C}_{6} \mathrm{D}_{6}$ at $\left.7.15 \mathrm{ppm}\right)$. Data are reported as: multiplicity $(\mathrm{ap}=$ apparent, $\mathrm{br}=$ broad, $\mathrm{s}=$ singlet, $\mathrm{d}=$ doublet, $\mathrm{t}=$ triplet, $\mathrm{q}=$ quartet, $\mathrm{m}=$ multiplet), integration and coupling constant(s) in Hz. ${ }^{13} \mathrm{C}$ NMR spectra were recorded on a Bruker Avance 300 $(75 \mathrm{MHz})$ or Avance400 (100 MHz) spectrometer. Chemical shifts are reported in ppm from tetramethylsilane, with the solvent resonance employed as the internal standard $\left(\mathrm{CDCl}_{3}\right.$ at $77.0 \mathrm{ppm}$ or $\mathrm{C}_{6} \mathrm{D}_{6}$ at $\left.128.02 \mathrm{ppm}\right)$. High-resolution mass spectra were recorded on a Kratos-Concept IIH instrument operated by the OttawaCarleton Mass Spectrometry Centre.

Materials. Dichloromethane and acetonitrile were dried by distillation over calcium hydride. Tetrahydrofuran was dried by distillation over sodium/benzophenone ketyl. Diethyl ether was purified by passage through a column of activated alumina under a nitrogen atmosphere. ${ }^{3}$ Unless otherwise noted, all

(1) For specifications, see http://www.luzchem.com/handbook/LESUVA011.pdf

(2) Alternatively, this apparatus could be substituted for a medium pressure Hg lamp fitted with a uranium glass filter.

(3) Pangborn, A. B.; Giardello, M. A.; Grubbs, R. H.; Rosen, R. K.; Timmers, F. J. Organometallics 1996, $15,1518$. 
commercial materials were used without further purification. Reagents were purchased from Sigma-Aldrich. Cyclohept-2-enone, ${ }^{4}$ cyclooct-2-enone, ${ }^{5}$ 2,6-cycloheptadienone, ${ }^{6}$ 2,3-benzo-2,6-cycloheptadienone ${ }^{7}$ and 4acetylcyclohept-2-enone ${ }^{8}$ were prepared according to literature procedures.

General Procedure for Solvent Scan (Table 1). Into each of 10 borosilicate tubes equipped with stir bars were added cyclohept-2-enone $(0.025 \mathrm{~g}, 0.23 \mathrm{mmol})$ and benzimidazole $(80 \mathrm{mg}, 0.68 \mathrm{mmol})$. To each tube was added $4 \mathrm{~mL}$ of solvent (Table 1). The tube was capped with a rubber septum and purged with a nitrogen balloon and an outlet for 10 minutes with stirring. The tubes were then placed on a Luzchem rotating carousel in a Rayonet photoreactor equipped with eight $8 \mathrm{~W}$ UV-A bulbs for 16 hours. The reactions were monitored by TLC (5\% $\mathrm{MeOH} / \mathrm{CH}_{2} \mathrm{Cl}_{2}$ ). The contents of each tube were then transferred to round-bottom flasks, and the solvent was removed under reduced pressure. The contents of the flask were then completely dissolved in $1.00 \mathrm{~mL}$ of $d_{6^{-}}$ DMSO. ${ }^{1} \mathrm{H}$ NMR spectra of these solutions were recorded, and the conversion calculated based on the relative integration of the resonance corresponding to one of the product's protons $(1 \mathrm{H})$ at $8.32 \mathrm{ppm}$ compared to the integration of the resonance corresponding to a benzimidazole peak at $8.24 \mathrm{ppm}(1 \mathrm{H}) .^{9}$ This method is estimated to have an error of approximately $\pm 5 \%$.

General Procedure for Photoinduced 1,4-Addition of Nitrogen Heterocycles (Table 2 and Table 3). A borosilicate tube was charged with a stir bar, cycloalkenone $(0.45 \mathrm{mmol})$ and nitrogen heterocycle $(1.38 \mathrm{mmol})$. A volume of $8.0 \mathrm{~mL}$ dichloromethane and $1.0 \mathrm{~mL}$ acetonitrile was added to the mixture. The tube was capped with a septum and was purged with a nitrogen balloon and an outlet for 5 minutes while stirring. The tube was then placed between two Luzchem exposure panels each equipped with four ultraviolet lamps for 18 hours. The reaction was monitored by thin layer chromatography. The crude reaction mixture was concentrated under reduced pressure and purified by silica gel chromatography to give the 1,4-addition product.

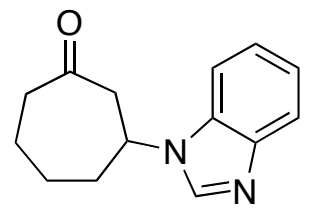

3-Benzoimidazol-1-yl-cycloheptanone (3a)(table 2, entry 1). Isolated $97 \mathrm{mg}(94 \%)$ as a clear, colorless oil after column chromatography $\left(4 \% \mathrm{MeOH} / \mathrm{CH}_{2} \mathrm{Cl}_{2}\right)$. TLC $R_{\mathrm{f}} 0.56\left(10 \% \mathrm{MeOH} / \mathrm{CH}_{2} \mathrm{Cl}_{2}\right) ;{ }^{1} \mathrm{H} \mathrm{NMR}\left(\mathrm{CDCl}_{3}, 300\right.$ $\mathrm{MHz}) 7.96(\mathrm{~s}, 1 \mathrm{H}), 7.80-7.74(\mathrm{~m}, 1 \mathrm{H}), 7.42-7.34(\mathrm{~m}, 1 \mathrm{H}), 7.30-7.22(\mathrm{~m}, 2 \mathrm{H}), 4.51(\mathrm{tt}, 1 \mathrm{H}, J=11.5 \mathrm{and} 2.9 \mathrm{~Hz})$, $3.27(\mathrm{dd}, 1 \mathrm{H}, J=13.8$ and $11.8 \mathrm{~Hz}), 2.86(\mathrm{dt}, 1 \mathrm{H}, J=13.8$ and $2.4 \mathrm{~Hz}), 2.70-2.61(\mathrm{~m}, 1 \mathrm{H}), 2.54(\mathrm{ddd}, 1 \mathrm{H}, J=$ 16.0, 11.7 and $4.1 \mathrm{~Hz}), 2.36-2.02(\mathrm{~m}, 4 \mathrm{H}), 1.86-1.46(\mathrm{~m}, 2 \mathrm{H}) ;{ }^{13} \mathrm{C}$ NMR $(75 \mathrm{MHz}) 209.8,144.1,140.6,133.0$, 123.5, 122.9, 120.9, 110.4, 53.9, 50.3, 44.3, 37.8, 27.3, 24.1; IR (film): 3383, 3086, 3054, 2935, 2862, 1700, 1613, 1489, 1458, 1406, 1348, 1279, 1219, 1009, 935, 889, 744, 700, $633 \mathrm{~cm}^{-1}$; HRMS (EI): Exact mass calcd for $\mathrm{C}_{14} \mathrm{H}_{16} \mathrm{~N}_{2} \mathrm{O}[\mathrm{M}]^{+}: 228.1263$. Found: 228.1261 .

(4) Mihelich, E. D.; Eickhoff, D. J. J. Org. Chem. 1983, 48, 4135.

(5) Chidambaram, N.; Chandrasekaran, S. J. Org. Chem. 1987, 52, 5048.

(6) Nicolaou, K. C.; Montagnon, T.; Baran, P. S.; Zhong, Y.-L. J. Am. Chem. Soc. 2002, 124, 2245.

(7) Hart, H.; Dunkelblum, E. J. Am. Chem. Soc. 1978, 100, 5141.

(8) Fujimoto, Y.; Xie, R.; Tully, S. E.; Berova, N.; Nakanishi, K. Chirality 2002, 14, 340.

(9) Benzimidazole was used as the internal standard for this calculation rather than 2-cycloheptenone, which is known to undergo photochemical dimerization. 


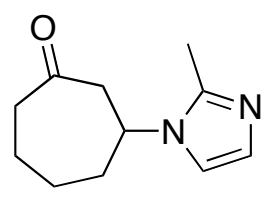

3-(2-Methyl-imidazol-1-yl)-cycloheptanone (3b)(table 2, entry 2). Isolated $61 \mathrm{mg}(70 \%)$ as a clear, colorless

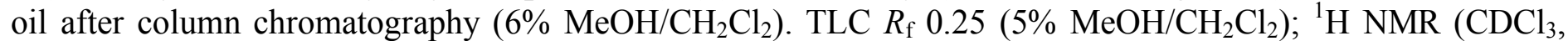
$300 \mathrm{MHz}) \delta 6.89($ ap s, $1 \mathrm{H}), 6.82(\mathrm{~d}, 1 \mathrm{H}, J=1.1 \mathrm{~Hz}), 4.16(\mathrm{tt}, 1 \mathrm{H}, J=11.6$ and $2.8 \mathrm{~Hz}), 3.07(\mathrm{dd}, 1 \mathrm{H}, J=13.8$ and $11.7 \mathrm{~Hz}), 2.67(\mathrm{dt}, 1 \mathrm{H}, J=2.4$ and $13.7 \mathrm{~Hz}), 2.62-2.42(\mathrm{~m}, 2 \mathrm{H}), 2.37(\mathrm{~s}, 3 \mathrm{H}), 2.19-1.96(\mathrm{~m}, 3 \mathrm{H}), 1.90(\mathrm{q}$, $1 \mathrm{H}, J=11.2 \mathrm{~Hz}), 1.72$ (ddd, $1 \mathrm{H}, J=16.1,12.5$ and $4.4 \mathrm{~Hz}), 1.56-1.38(\mathrm{~m}, 1 \mathrm{H}) ;{ }^{13} \mathrm{C}$ NMR $(75 \mathrm{MHz}) 209.9$, 143.8, 128.0, 115.4, 53.6, 51.1, 44.2, 38.8, 27.1, 24.0, 13.6; IR (film): 3376, 3110, 2934, 2862, 1698, 1447, 1419, 1279, 749, $678 \mathrm{~cm}^{-1}$; HRMS (EI): Exact mass calcd for $\mathrm{C}_{11} \mathrm{H}_{16} \mathrm{~N}_{2} \mathrm{O}[\mathrm{M}]^{+}: 192.1263$. Found: 192.1276.

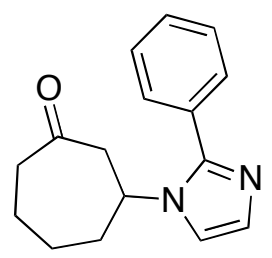

3-(2-Phenyl-imidazol-1-yl)-cycloheptanone (3c)(table 2, entry 3). Isolated $51 \mathrm{mg}(44 \%)$ as a white crystalline solid after column chromatography $\left(5 \% \mathrm{MeOH} / \mathrm{CH}_{2} \mathrm{Cl}_{2}\right)$. TLC $R_{\mathrm{f}} 0.53\left(10 \% \mathrm{MeOH} / \mathrm{CH}_{2} \mathrm{Cl}_{2}\right) ;{ }^{1} \mathrm{H} \mathrm{NMR}\left(\mathrm{CDCl}_{3}\right.$, $300 \mathrm{MHz}) 7.54-7.38(\mathrm{~m}, 5 \mathrm{H}), 7.14(\mathrm{~d}, 1 \mathrm{H}, J=0.9 \mathrm{~Hz}), 7.05(\mathrm{~d}, 1 \mathrm{H}, J=1.3 \mathrm{~Hz}), 4.48(\mathrm{tt}, 1 \mathrm{H}, J=11.3$ and 2.8 $\mathrm{Hz}), 3.16(\mathrm{dd}, 1 \mathrm{H}, J=14.3$ and $11.6 \mathrm{~Hz}), 2.78(\mathrm{dt}, 1 \mathrm{H}, J=14.3$ and $2.5 \mathrm{~Hz}), 2.63-2.54(\mathrm{~m}, 1 \mathrm{H}), 2.39(\mathrm{ddd}, 1 \mathrm{H}$, $J=16.0,12.1$ and $3.8 \mathrm{~Hz}), 2.24-2.13(\mathrm{~m}, 1 \mathrm{H}), 2.08-1.91(\mathrm{~m}, 3 \mathrm{H}), 1.79-1.61(\mathrm{~m}, 1 \mathrm{H}), 1.47-1.29(\mathrm{~m}, 1 \mathrm{H}) ;{ }^{13} \mathrm{C}$ NMR (75 MHz) 209.8, 147.3, 130.8, 129.7, 129.5, 129.4, 129.2, 116.7, 53.8, 51.7, 44.1, 39.5, 27.2, 24.1; IR (film): 3380, 2934, 2862, 1700, 1466, 1445, 1416, 1275, 1260, 765, 749, 714, $701 \mathrm{~cm}^{-1}$; HRMS (EI): Exact mass calcd for $\mathrm{C}_{16} \mathrm{H}_{18} \mathrm{~N}_{2} \mathrm{O}[\mathrm{M}]^{+}: 254.1419$. Found: 254.1443.

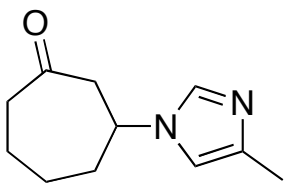

7

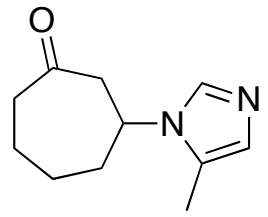

1

3-(4-Methyl-imidazol-1-yl)-cycloheptanone (major) and 3-(5-methyl-imidazol-1-yl)-cycloheptanone (minor) (3d)(table 2, entry 4). ${ }^{10}$ Isolated $64 \mathrm{mg}(73 \%)$ as a yellow oil after column chromatography $(1.5 \%$ $\left.\mathrm{MeOH} / \mathrm{CH}_{2} \mathrm{Cl}_{2}\right)$. This mixture of isomers co-eluted in a variety of solvent systems. TLC $R_{\mathrm{f}} 0.25(5 \%$ $\mathrm{MeOH} / \mathrm{CH}_{2} \mathrm{Cl}_{2}$ ); IR (film, mixture): 3374, 3111, 2935, 2863, 1699, 1496, 1448, 1275, 1260, 1233, 1164, 764, $749 \mathrm{~cm}^{-1}$; HRMS (EI): Exact mass calcd for $\mathrm{C}_{11} \mathrm{H}_{16} \mathrm{~N}_{2} \mathrm{O}[\mathrm{M}]^{+}:$192.1263. Found: 192.1268.

(10) The regioisomers were differentiated by NOE measurements and were consistent with the expected reactivity. 

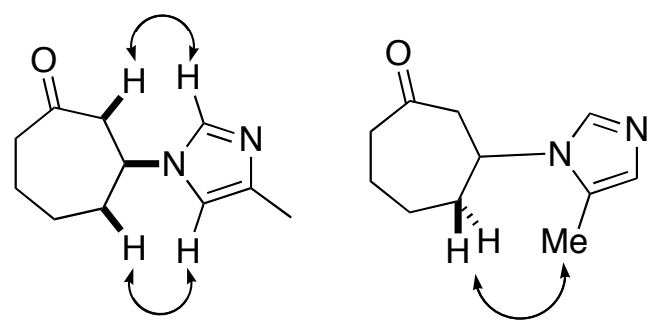

major product: ${ }^{1} \mathrm{H}$ NMR $\left(\mathrm{CDCl}_{3}, 300 \mathrm{MHz}\right) 7.39(\mathrm{~s}, 1 \mathrm{H}), 6.61(\mathrm{~s}, 1 \mathrm{H}), 4.19(\mathrm{tt}, 1 \mathrm{H}, J=11.3$ and 2.9 $\mathrm{Hz}), 3.08(\mathrm{dd}, 1 \mathrm{H}, J=14.0$ and $11.6 \mathrm{~Hz}), 2.76(\mathrm{dt}, 1 \mathrm{H}, J=4.8$ and $2.6 \mathrm{~Hz}), 2.59(\mathrm{dt}, 1 \mathrm{H}, J=9.5 \mathrm{and} 4.4 \mathrm{~Hz})$, $2.47(\mathrm{ddd}, 1 \mathrm{H}, J=15.7,11.5$ and $3.9 \mathrm{~Hz}), 2.17(\mathrm{~s}, 3 \mathrm{H}), 2.09-1.86(\mathrm{~m}, 4 \mathrm{H}), 1.79-1.61(\mathrm{~m}, 1 \mathrm{H}), 1.57-1.39(\mathrm{~m}$, $1 \mathrm{H}) ;{ }^{13} \mathrm{C}$ NMR (75 MHz) 210.0, 139.0, 134.7, 113.5, 54.9, 51.5, 44.3, 39.1, 27.0, 24.0, 14.1 .

minor product: ${ }^{1} \mathrm{H} \mathrm{NMR}\left(\mathrm{CDCl}_{3}, 300 \mathrm{MHz}\right.$, diagnostic peaks only) $7.45(\mathrm{~s}, 1 \mathrm{H}), 6.73(\mathrm{~s}, 1 \mathrm{H}), 4.08(\mathrm{tt}$, $1 \mathrm{H}, J=11.7$ and $2.6 \mathrm{~Hz}), 2.18(\mathrm{~s}, 3 \mathrm{H}) ;{ }^{13} \mathrm{C} \mathrm{NMR}(75 \mathrm{MHz}) 209.9,134.0,127.2,126.7,52.9,51.3,38.9,27.3$, 23.9, 9.9.

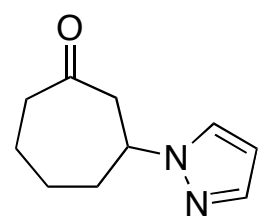

3-Pyrazol-1-yl-cycloheptanone (3e)(table 2, entry 5). Isolated $81 \mathrm{mg}(99 \%)$ as a clear, colorless oil after column chromatography $\left(4 \% \mathrm{MeOH} / \mathrm{CH}_{2} \mathrm{Cl}_{2}\right)$. TLC $R_{\mathrm{f}} 0.64\left(10 \% \mathrm{MeOH} / \mathrm{CH}_{2} \mathrm{Cl}_{2}\right) ;{ }^{1} \mathrm{H} \mathrm{NMR}\left(\mathrm{CDCl}_{3}, 300\right.$ $\mathrm{MHz}) 7.48(\mathrm{~d}, 1 \mathrm{H}, J=1.5 \mathrm{~Hz}), 7.37(\mathrm{~d}, 1 \mathrm{H}, J=2.3 \mathrm{~Hz}), 6.21(\mathrm{t}, 1 \mathrm{H}, J=2.1 \mathrm{~Hz}), 4.46(\mathrm{tt}, 1 \mathrm{H}, J=11.1$ and 3.2 $\mathrm{Hz}), 3.25(\mathrm{dd}, 1 \mathrm{H}, J=14.4$ and $11.2 \mathrm{~Hz}), 2.82(\mathrm{ddd}, 1 \mathrm{H}, J=14.4,2.9$ and $1.8 \mathrm{~Hz}), 2.61(\mathrm{dddd}, 1 \mathrm{H}, J=16.0$, 5.3, 4.0 and $1.3 \mathrm{~Hz}), 2.48(\mathrm{ddd}, 1 \mathrm{H}, J=15.9,11.2$ and $3.9 \mathrm{~Hz}), 2.25-1.90(\mathrm{~m}, 4 \mathrm{H}), 1.82-1.67(\mathrm{~m}, 1 \mathrm{H}), 1.59-1.45$ $(\mathrm{m}, 1 \mathrm{H}) ;{ }^{13} \mathrm{C}$ NMR $(75 \mathrm{MHz}) 210.4,139.1,127.0,105.3,58.7,50.1,44.0,37.7,26.5,23.6$; IR (film): 2935, 2861, 1702, 1511, 1445, 1397, 1284, 1225, 1090, 1048, 967, 913, 747, $623 \mathrm{~cm}^{-1}$; HRMS (EI): Exact mass calcd for $\mathrm{C}_{10} \mathrm{H}_{14} \mathrm{~N}_{2} \mathrm{O}[\mathrm{M}]^{+}:$178.1106. Found: 178.1094.

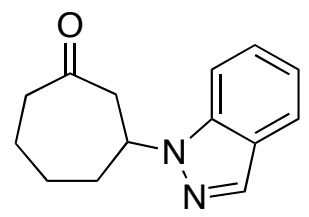

3-Indazol-1-yl-cycloheptanone (3f)(table 2, entry 6). Isolated $95 \mathrm{mg}(92 \%)$ as a clear, colorless oil after column chromatography (40\% EtOAc/hexanes). TLC $R_{\mathrm{f}} 0.37$ (40\% EtOAc/hexanes); ${ }^{1} \mathrm{H} \mathrm{NMR}\left(\mathrm{CDCl}_{3}, 300\right.$ $\mathrm{MHz}) 7.06(\mathrm{~d}, 1 \mathrm{H}, J=5.7 \mathrm{~Hz}), 6.81($ ap ddd, $2 \mathrm{H}, J=13.6,10.2$ and $7.0 \mathrm{~Hz}), 6.43(\mathrm{dd}, 1 \mathrm{H}, J=14.0$ and $6.8 \mathrm{~Hz})$, $6.22(\mathrm{dd}, 1 \mathrm{H}, J=13.8$ and $6.9 \mathrm{~Hz}), 3.89-3.86(\mathrm{~m}, 1 \mathrm{H}), 2.66-2.55(\mathrm{~m}, 1 \mathrm{H}), 2.09(\mathrm{br} \mathrm{d}, J=14.4 \mathrm{~Hz}), 1.87-1.62$ $(\mathrm{m}, 2 \mathrm{H}), 1.49-1.41(\mathrm{~m}, 2 \mathrm{H}), 1.24-1.13(\mathrm{~m}, 2 \mathrm{H}), 1.04-0.89(\mathrm{~m}, 1 \mathrm{H}), 0.80-0.65(\mathrm{~m}, 1 \mathrm{H}) ;{ }^{13} \mathrm{C} \mathrm{NMR}(75 \mathrm{MHz})$ 209.8, 148.5, 125.9, 121.7, 121.3, 120.7, 120.1, 117.3, 60.4, 50.3, 43.9, 38.1, 26.5, 23.5; IR (film): 3118, 3059, 2934, 2861, 1701, 1628, 1513, 1447, 1379, 1348, 1291, 1135, 912, 757, $655 \mathrm{~cm}^{-1}$; HRMS (EI): Exact mass calcd for $\mathrm{C}_{14} \mathrm{H}_{16} \mathrm{~N}_{2} \mathrm{O}[\mathrm{M}]^{+}: 228.1263$. Found: 228.1267 . 


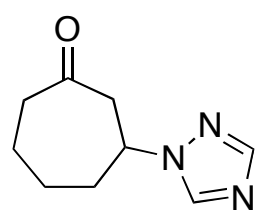

3-[1,2,4] Triazol-1-yl-cycloheptanone (3g)(table 2, entry 7). Isolated $54 \mathrm{mg}(66 \%)$ as a clear, colorless oil after column chromatography $\left(3 \% \mathrm{MeOH} / \mathrm{CH}_{2} \mathrm{Cl}_{2}\right)$. TLC $R_{\mathrm{f}} 0.46\left(10 \% \mathrm{MeOH} / \mathrm{CH}_{2} \mathrm{Cl}_{2}\right) ;{ }^{1} \mathrm{H}$ NMR $\left(\mathrm{CDCl}_{3}, 300\right.$ $\mathrm{MHz}) 8.09(\mathrm{~s}, 1 \mathrm{H}), 7.93(\mathrm{~s}, 1 \mathrm{H}), 4.55(\mathrm{tt}, 1 \mathrm{H}, J=11.3$ and $3.3 \mathrm{~Hz}), 3.30(\mathrm{dd}, 1 \mathrm{H}, J=14.2$ and $11.2 \mathrm{~Hz}), 2.85$ (ddd, $1 \mathrm{H}, J=14.2,2.8$ and $1.7 \mathrm{~Hz}), 2.71-2.62(\mathrm{~m}, 1 \mathrm{H}), 2.51(\mathrm{ddd}, 1 \mathrm{H}, J=16.1,11.2$ and $4.0 \mathrm{~Hz}), 2.29-1.94(\mathrm{~m}$, 4H), 1.87-1.72 (m, 1H), 1.63-1.50 (m, 1H); ${ }^{13} \mathrm{C}$ NMR (75 MHz) 209.8, 152.3, 141.7, 57.5, 50.0, 44.4, 37.9, 26.6, 23.8; IR (film): 3165, 2936, 2878, 1702, 1503, 1445, 1347, 1275, 1140, 1013, 913, 744, $681,663 \mathrm{~cm}^{-1}$; HRMS (EI): Exact mass calcd for $\mathrm{C}_{9} \mathrm{H}_{13} \mathrm{~N}_{3} \mathrm{O}[\mathrm{M}]^{+}:$179.1059. Found: 179.1062 .

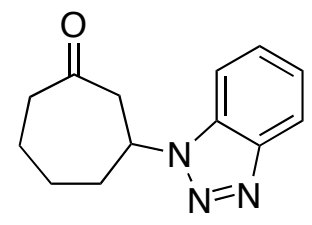

3-Benzotriazol-1-yl-cycloheptanone (3h)(table 2, entry 8). Isolated $56 \mathrm{mg}(53 \%)$ as a white crystalline solid after column chromatography $\left(1.5 \% \mathrm{MeOH} / \mathrm{CH}_{2} \mathrm{Cl}_{2}\right)$. TLC $R_{\mathrm{f}} 0.55\left(10 \% \mathrm{MeOH} / \mathrm{CH}_{2} \mathrm{Cl}_{2}\right) ;{ }^{1} \mathrm{H} \mathrm{NMR}\left(\mathrm{CDCl}_{3}\right.$, $300 \mathrm{MHz}) 8.05(\mathrm{dt}, 1 \mathrm{H}, J=8.3$ and $1.0 \mathrm{~Hz}$ ), $7.53(\mathrm{ddd}, 1 \mathrm{H}, J=8.4,1.3$ and $0.9 \mathrm{~Hz}), 7.45(\mathrm{ddd}, 1 \mathrm{H}, J=8.2,6.6$ and $1.0 \mathrm{~Hz}$ ), 7.34 (ddd, $1 \mathrm{H}, J=8.3,6.6$ and $1.4 \mathrm{~Hz}), 4.97(\mathrm{tt}, 1 \mathrm{H}, J=10.7$ and $3.2 \mathrm{~Hz}), 3.59(\mathrm{dd}, 1 \mathrm{H}, J=14.2$ and $11.4 \mathrm{~Hz}$ ), $2.92(\mathrm{ddd}, 1 \mathrm{H}, J=14.2,3.0$ and $1.9 \mathrm{~Hz}$ ), 2.73 (dddd, 1H, $J=16.2,5.5,4.2$ and $1.4 \mathrm{~Hz}$ ), 2.58 (ddd, $1 \mathrm{H}, J=16.1,11.2$ and $4.1 \mathrm{~Hz}), 2.50-2.35,(\mathrm{~m}, 1 \mathrm{H}), 2.35-2.25(\mathrm{~m}, 1 \mathrm{H}), 2.15-1.77(\mathrm{~m}, 4 \mathrm{H}), 1.67-1.51(\mathrm{~m}$, 1H); ${ }^{13} \mathrm{C}$ NMR (75 MHz) 210.1, 146.4, 132.2, 127.8, 124.5, 120.6, 109.7, 56.7, 49.7, 44.4, 37.4, 26.9, 24.0; IR (film): 3063, 2934, 2861, 1702, 1453, 1299, 1269, 1237, 1162, 1069, 766, $749 \mathrm{~cm}^{-1}$; HRMS (EI): Exact mass calcd for $\mathrm{C}_{13} \mathrm{H}_{15} \mathrm{~N}_{3} \mathrm{O}[\mathrm{M}]^{+}: 229.1215$. Found: 229.1210 .

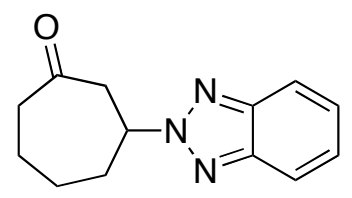

3-Benzotriazol-2-yl-cycloheptanone (3h)(table 2, entry 8). Isolated $12 \mathrm{mg}(11 \%)$ as a white crystalline solid after column chromatography $\left(1.5 \% \mathrm{MeOH} / \mathrm{CH}_{2} \mathrm{Cl}_{2}\right)$. TLC $R_{\mathrm{f}} 0.55\left(10 \% \mathrm{MeOH} / \mathrm{CH}_{2} \mathrm{Cl}_{2}\right) ;{ }^{1} \mathrm{H} \mathrm{NMR}\left(\mathrm{CDCl}_{3}\right.$, $300 \mathrm{MHz}) 7.85(\mathrm{dd}, 2 \mathrm{H}, J=6.6$ and $3.0 \mathrm{~Hz}), 7.38(\mathrm{dd}, 2 \mathrm{H}, J=6.6$ and $3.1 \mathrm{~Hz}), 5.24-5.13(\mathrm{~m}, 1 \mathrm{H}), 3.54(\mathrm{dd}, 1 \mathrm{H}$, $J=15.1$ and $10.8 \mathrm{~Hz}), 3.10(\mathrm{dd}, 1 \mathrm{H}, J=15.2$ and $2.5 \mathrm{~Hz}), 2.76-2.65(\mathrm{~m}, 1 \mathrm{H}), 2.59(\mathrm{ddd}, 1 \mathrm{H}, J=15.9,10.4$ and $3.6 \mathrm{~Hz}), 2.47-2.38(\mathrm{~m}, 2 \mathrm{H}), 2.09-1.93(\mathrm{~m}, 2 \mathrm{H}), 1.92-1.6(\mathrm{~m}, 2 \mathrm{H}) ;{ }^{13} \mathrm{C}$ NMR $(75 \mathrm{MHz}) 209.9,144.4,126.9$, 118.5, 63.7, 49.7, 44.6, 37.9, 27.0, 24.1; IR (film): 3502, 3388, 3065, 2933, 2861, 1701, 1447, 1346, 1319, 1295, 1272, 1241, 1212, 1199, 1142, $749 \mathrm{~cm}^{-1}$; HRMS (EI): Exact mass calcd for $\mathrm{C}_{13} \mathrm{H}_{15} \mathrm{~N}_{3} \mathrm{O}[\mathrm{M}]^{+}: 229.1215$. Found: 229.1198. 


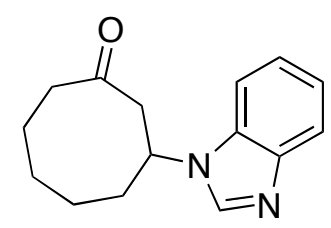

3-Benzoimidazol-1-yl-cyclooctanone (12)(table 3, entry 2). Isolated $58 \mathrm{mg}(90 \%)$ as a clear, colorless oil after column chromatography $\left(4 \% \mathrm{MeOH} / \mathrm{CH}_{2} \mathrm{Cl}_{2}\right)$. TLC $R_{\mathrm{f}} 0.69\left(10 \% \mathrm{MeOH} / \mathrm{CH}_{2} \mathrm{Cl}_{2}\right) ;{ }^{1} \mathrm{H} \mathrm{NMR}\left(\mathrm{CDCl}_{3}, 300\right.$ $\mathrm{MHz}) 8.03(\mathrm{~s}, 1 \mathrm{H}), 7.82-7.79(\mathrm{~m}, 1 \mathrm{H}), 7.52-7.49(\mathrm{~m}, 1 \mathrm{H}), 7.35-7.26(\mathrm{~m}, 2 \mathrm{H}), 4.67(\mathrm{tt}, 1 \mathrm{H}, J=11.9$ and $3.7 \mathrm{~Hz})$, $3.35(\mathrm{t}, 1 \mathrm{H}, J=11.9 \mathrm{~Hz}), 2.71(\mathrm{ddd}, 1 \mathrm{H}, J=11.8,3.4$ and $1.6 \mathrm{~Hz}), 2.53$ (ap ddd, $2 \mathrm{H}, J=5.6,5.4$ and $2.8 \mathrm{~Hz}$ ), 2.27-1.22 (m, 8H); ${ }^{13} \mathrm{C}$ NMR (75 MHz) 212.1, 143.7, 140.7, 133.1, 123.6, 123.0, 120.8, 110.4, 56.2, 46.6, 44.3, 33.3, 28.1, 23.5, 22.7; IR (film): 2936, 2872, 1699, 1489, 1458, 1283, 1221, 1206, 1082, $744 \mathrm{~cm}^{-1}$; HRMS (EI): Exact mass calcd for $\mathrm{C}_{15} \mathrm{H}_{18} \mathrm{~N}_{2} \mathrm{O}[\mathrm{M}]^{+}: 242.1419$. Found: 242.1428 .

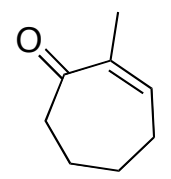

2-Methyl-cyclohept-2-enone (13)(table 3, entry 3). Prepared according to a modification of the procedure of Paquette. ${ }^{11}$ To a stirred solution of triethylamine $(4.86 \mathrm{~g}, 48 \mathrm{mmol})$ in $8 \mathrm{~mL}$ dry dimethylformamide (both components freshly distilled from calcium hydride) were added chlorotrimethylsilane (2.61 g, $24 \mathrm{mmol})$ and cyclohexanone (1.96 g, $20 \mathrm{mmol})$. This mixture was heated at the reflux temperature for $6 \mathrm{~h}$, cooled to room temperature, and diluted with $30 \mathrm{~mL}$ pentane. The precipitated triethylamine hydrochloride was separated by filtration and washed well with pentane $(3 \times 15 \mathrm{~mL})$. The combined filtrates were washed with ice-cold saturated sodium bicarbonate solution $(3 \times 30 \mathrm{~mL})$ and brine $(10 \mathrm{~mL})$ prior to drying over $\mathrm{Na}_{2} \mathrm{SO}_{4}$. Solvent evaporation left $3.04 \mathrm{~g} \mathrm{(89 \% )}$ of the silyl enol ether that was used directly without further purification.

$n$-Butyllithium in hexane $(46 \mathrm{~mL}$ of $1.10 \mathrm{M}, 51 \mathrm{mmol}$ ) was slowly added under nitrogen during $6 \mathrm{~h}$ to a cold ($\left.40{ }^{\circ} \mathrm{C}\right)$, magnetically stirred solution of the preceding product $(3.04 \mathrm{~g}, 17.8 \mathrm{mmol})$ and 1,1-dichloroethane (5.64 $\mathrm{g}, 57.0 \mathrm{mmol})$ in anhydrous ether $(7.0 \mathrm{~mL})$. The mixture was allowed to warm to $0{ }^{\circ} \mathrm{C}$ over $1 \mathrm{~h}$, at which point it was diluted with ether $(15 \mathrm{~mL})$, washed with water $(4 \times 10 \mathrm{~mL})$, dried, and concentrated. The residual oil $(4.0 \mathrm{~g})$ was dissolved in a mixture of toluene $(80 \mathrm{~mL})$ and ethylene glycol $(7.5 \mathrm{~mL})$ and heated at the reflux temperature under nitrogen for $24 \mathrm{~h}$. The crude reaction mixture was concentrated under reduced pressure. The oil was taken up in hexanes and washed with water $(3 \times 30 \mathrm{~mL})$ to remove ethylene glycol, followed by drying over $\mathrm{Na}_{2} \mathrm{SO}_{4}$. The mixture was then submitted twice to column chromatography $(8 \%$ EtOAc/Hexanes, then $\left.\mathrm{CH}_{2} \mathrm{Cl}_{2}\right)$ to give the product $(0.483 \mathrm{~g}, 20 \%)$ as a clear, colourless oil. TLC $R_{\mathrm{f}} 0.32(8 \%$ EtOAc/Hexanes $) ;{ }^{1} \mathrm{H}$ $\operatorname{NMR}\left(\mathrm{CDCl}_{3}, 300 \mathrm{MHz}\right) 6.49(\mathrm{tq}, 1 \mathrm{H}, J=6.4$ and $1.4 \mathrm{~Hz}), 2.50($ ap dd, $2 \mathrm{H}, J=7.4$ and $5.6 \mathrm{~Hz}), 2.32-2.26(\mathrm{~m}$, 2H), 1.75 (ap q, 3H, $J=1.4 \mathrm{~Hz}), 1.71-1.63(\mathrm{~m}, 4 \mathrm{H}) ;{ }^{13} \mathrm{C} \mathrm{NMR}(75 \mathrm{MHz}) 205.2,142.9,139.6,42.7,27.9,25.5$, 21.7, 19.7; IR (film): 2938, 2866, 1667, 1453, $1375 \mathrm{~cm}^{-1}$; HRMS (EI): Exact mass calcd for $\mathrm{C}_{8} \mathrm{H}_{12} \mathrm{O}[\mathrm{M}]^{+}$: 124.0888. Found: 124.0886 .

(11) Paquette, L. A.; Hun Ham, W. J. Am. Chem. Soc. 1987, 109, 3025. 


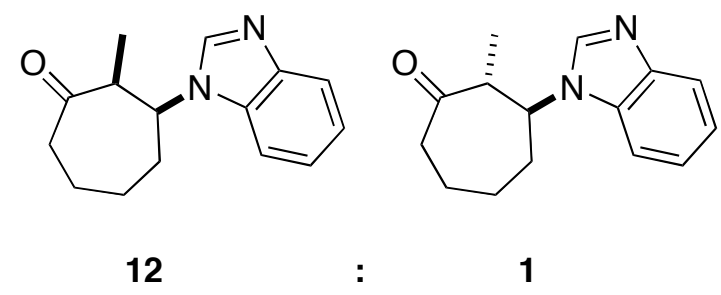

syn 3-Benzoimidazol-1-yl-2-methyl-cycloheptanone (14)(table 3, entry 3). ${ }^{\mathbf{1 2}}$ Isolated $71 \mathrm{mg}(66 \%)$ as a clear, colourless oil after column chromatography $\left(3 \% \mathrm{MeOH} / \mathrm{CH}_{2} \mathrm{Cl}_{2}\right)$. TLC $\left.R_{\mathrm{f}} 0.32\left(3 \% \mathrm{MeOH} / \mathrm{CH}_{2} \mathrm{Cl}\right)_{2}\right){ }^{1} \mathrm{H}$ $\operatorname{NMR}\left(\mathrm{CDCl}_{3}, 300 \mathrm{MHz}\right) 8.03(\mathrm{~s}, 1 \mathrm{H}), 7.82(\mathrm{ddd}, 1 \mathrm{H}, J=3.5,2.2$ and $0.6 \mathrm{~Hz}), 7.42-7.39(\mathrm{~m}, 1 \mathrm{H}), 7.35-7.27(\mathrm{~m}$, $2 \mathrm{H}), 4.91(\mathrm{ddd}, 1 \mathrm{H}, J=9.0,6.7$ and $5.0 \mathrm{~Hz}), 3.18(\mathrm{qd}, 1 \mathrm{H}, J=7.1$ and $5.0 \mathrm{~Hz}), 2.81-2.62(\mathrm{~m}, 2 \mathrm{H}), 2.34-2.27$ $(\mathrm{m}, 2 \mathrm{H}), 2.13-2.03(\mathrm{~m}, 2 \mathrm{H}), 1.86-1.71(\mathrm{~m}, 2 \mathrm{H}), 0.93(\mathrm{~d}, 3 \mathrm{H}, J=7.1 \mathrm{~Hz}) ;{ }^{13} \mathrm{C}$ NMR $(75 \mathrm{MHz}) 211.9,142.9$, 140.6, 133.1, 123.0, 122.4, 120.3, 109.5, 56.2, 50.0, 42.5, 31.2, 26.8, 24.1, 10.2; IR (film): 3084, 3059, 2936, 2864, 1700, 1488, 1458, 1284, 1218, $744 \mathrm{~cm}^{-1}$; HRMS (EI): Exact mass calcd for $\mathrm{C}_{15} \mathrm{H}_{18} \mathrm{~N}_{2} \mathrm{O}[\mathrm{M}]^{+}: 242.1419$. Found: 242.1405.

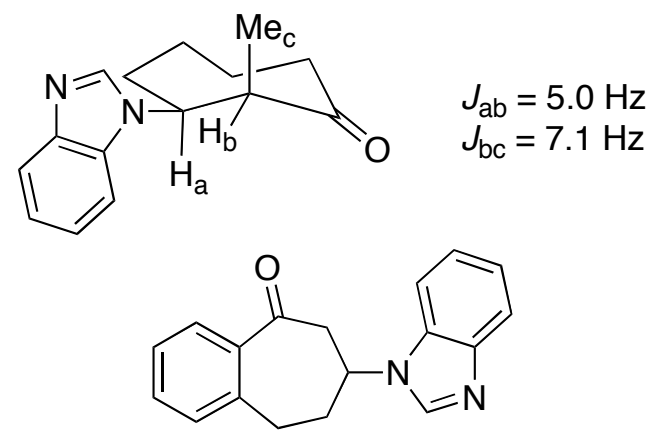

7-Benzoimidazol-1-yl-6,7,8,9-tetrahydro-benzocyclohepten-5-one (16)(table 3, entry 4). Isolated $115 \mathrm{mg}$ $(92 \%)$ as a clear, colourless oil after column chromatography $\left(3 \% \mathrm{MeOH} / \mathrm{CH}_{2} \mathrm{Cl}_{2}\right)$. TLC $R_{\mathrm{f}} 0.41(3 \%$ $\left.\mathrm{MeOH} / \mathrm{CH}_{2} \mathrm{Cl}_{2}\right) ;{ }^{1} \mathrm{H}$ NMR $\left(\mathrm{CDCl}_{3}, 300 \mathrm{MHz}\right) 8.02(\mathrm{~s}, 1 \mathrm{H}), 7.83-7.79(\mathrm{~m}, 2 \mathrm{H}), 7.52(\mathrm{td}, 1 \mathrm{H}, J=7.5 \mathrm{and} 1.5 \mathrm{~Hz})$, $7.41(\mathrm{dd}, 1 \mathrm{H}, J=7.7$ and $1.2 \mathrm{~Hz}), 7.37(\mathrm{dd}, 1 \mathrm{H}, J=3.7$ and $2.1 \mathrm{~Hz}), 7.32-7.28(\mathrm{~m}, 3 \mathrm{H}), 4.83(\mathrm{tdd}, 1 \mathrm{H}, J=10.1$, 6.8 and $3.8 \mathrm{~Hz}), 3.52(\mathrm{dd}, 1 \mathrm{H}, J=15.2$ and $10.0 \mathrm{~Hz}), 3.33-3.23(\mathrm{~m}, 2 \mathrm{H}), 3.07$ (ddd, $1 \mathrm{H}, J=15.7,5.7$ and 4.0 $\mathrm{Hz}), 2.58-2.34(\mathrm{~m}, 2 \mathrm{H}) ;{ }^{13} \mathrm{C}$ NMR $(75 \mathrm{MHz}) 200.0,143.7,140.5,140.1,137.7,132.9,132.8,130.0,128.9$, 127.3, 122.9, 122.4, 120.5, 109.8, 51.0, 47.0, 32.4, 30.7; IR (film): 3346, 3117, 3057, 2946, 2872, 1680, 1613, 1599, 1489, 1457, 1404, 1285, 1260, 1025, $745 \mathrm{~cm}^{-1}$; HRMS (EI): Exact mass calcd for $\mathrm{C}_{18} \mathrm{H}_{16} \mathrm{~N}_{2} \mathrm{O}[\mathrm{M}]^{+}$: 276.1263. Found: 276.1283.

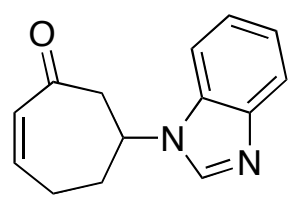

(12) The relative stereochemistry of the methyl and benzoimidazol-1-yl substituents was ascertained by analysis of the ${ }^{1} \mathrm{H}$ NMR spectroscopy vicinal coupling constants. Typical pseudo trans-diaxial coupling constants for $\mathrm{H}_{\mathrm{a}}(\approx 11.5 \mathrm{~Hz})$ were not observed. For examples of these coupling constants, see compounds $\mathbf{3 a}, \mathbf{3 b}, \mathbf{3 d}, \mathbf{3 e}, \mathbf{3 g}, \mathbf{3 h}, \mathbf{1 6}$. Rather, they are consistent with a cis relationship $\left(J_{\mathrm{ab}}=5.0 \mathrm{~Hz}\right)$. 
6-Benzoimidazol-1-yl-cyclohept-2-enone (18)(table 3, entry 5). Isolated $35 \mathrm{mg}(51 \%)$ as a clear, colourless oil after column chromatography $\left(4 \% \mathrm{MeOH} / \mathrm{CH}_{2} \mathrm{Cl}_{2}\right)$. TLC $R_{\mathrm{f}} 0.67\left(10 \% \mathrm{MeOH} / \mathrm{CH}_{2} \mathrm{Cl}_{2}\right) ;{ }^{1} \mathrm{H} \mathrm{NMR}\left(\mathrm{CDCl}_{3}\right.$, $300 \mathrm{MHz}) 8.01(\mathrm{~s}, 1 \mathrm{H}), 7.84-7.79(\mathrm{~m}, 1 \mathrm{H}), 7.41-7.27(\mathrm{~m}, 3 \mathrm{H}), 6.75(\mathrm{dt}, 1 \mathrm{H}, J=12.0$ and $5.4 \mathrm{~Hz}), 6.19$ (br d, $1 \mathrm{H}, J=12.2 \mathrm{~Hz}), 4.86(\mathrm{tt}, 1 \mathrm{H}, J=8.4$ and $5.2 \mathrm{~Hz}), 3.30(\mathrm{dd}, 1 \mathrm{H}, J=15.3$ and $8.4 \mathrm{~Hz}), 3.21(\mathrm{dd}, 1 \mathrm{H}, J=15.3$ and 5.4 Hz), 2.68-2.61 (m, 2H), 2.51-2.33 (m, 2H); ${ }^{13} \mathrm{C}$ NMR (75 MHz) 198.9, 146.8, 144.2, 140.7, 133.31, 133.27, 123.6, 123.0, 121.1, 110.2, 51.6, 49.6, 34.0, 27.9; IR (film): 2936, 1708, 1658, 1650, 1611, 1491, 1459, 1407, 1285, 1217, $745 \mathrm{~cm}^{-1}$; HRMS (EI): Exact mass calcd for $\mathrm{C}_{14} \mathrm{H}_{14} \mathrm{~N}_{2} \mathrm{O}[\mathrm{M}]^{+}:$226.1106. Found: 226.1095.
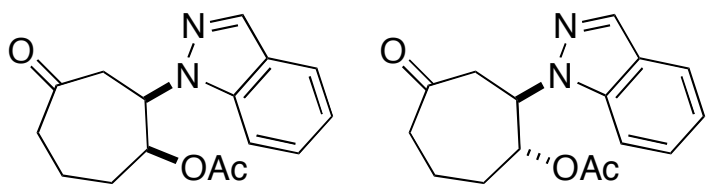

1.3

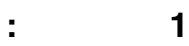

syn Acetic acid 2-indazol-1-yl-4-oxo-cycloheptyl ester (20)(table 3, entry 6). ${ }^{\mathbf{1 3}}$ Isolated $40 \mathrm{mg}(45 \%)$ as a clear, colourless oil after column chromatography (50\% EtOAc/Hex). TLC $R_{\mathrm{f}} 0.17\left(50 \%\right.$ EtOAc/Hexanes); ${ }^{1} \mathrm{H}$ NMR $\left(\mathrm{CDCl}_{3}, 300 \mathrm{MHz}\right) 7.91(\mathrm{~s}, 1 \mathrm{H}), 7.69(\mathrm{~d}, 1 \mathrm{H}, J=9.1 \mathrm{~Hz}), 7.66-7.63(\mathrm{~m}, 1 \mathrm{H}), 7.33-7.28(\mathrm{~m}, 1 \mathrm{H}), 7.13-$ $7.08(\mathrm{~m}, 1 \mathrm{H}), 5.43(\mathrm{dt}, 1 \mathrm{H}, J=7.1$ and $1.8 \mathrm{~Hz}), 5.16-5.11(\mathrm{~m}, 1 \mathrm{H}), 3.63(\mathrm{dd}, 1 \mathrm{H}, J=15.8$ and $10.6 \mathrm{~Hz}), 2.99$ $(\mathrm{dd}, 1 \mathrm{H}, J=15.9$ and $2.9 \mathrm{~Hz}), 2.92-2.83(\mathrm{~m}, 1 \mathrm{H}), 2.68-2.58(\mathrm{~m}, 1 \mathrm{H}), 2.30-2.17(\mathrm{~m}, 1 \mathrm{H}), 2.08(\mathrm{~s}, 3 \mathrm{H}), 2.01-1.77$ $(\mathrm{m}, 3 \mathrm{H}) ;{ }^{13} \mathrm{C}$ NMR (100 MHz) 169.4, 126.6, 122.3, 121.7, 120.3, 117.29, 117.26, 74.8, 61.6, 43.4, 31.1, 21.0, 18.4; IR (film): 2940, 1738, 1702, 1376, 1233, 1028, $859 \mathrm{~cm}^{-1}$; HRMS (EI): Exact mass calcd for $\mathrm{C}_{16} \mathrm{H}_{18} \mathrm{~N}_{2} \mathrm{O}_{3}$ $[\mathrm{M}]^{+}: 286.1317$. Found: 286.1325.
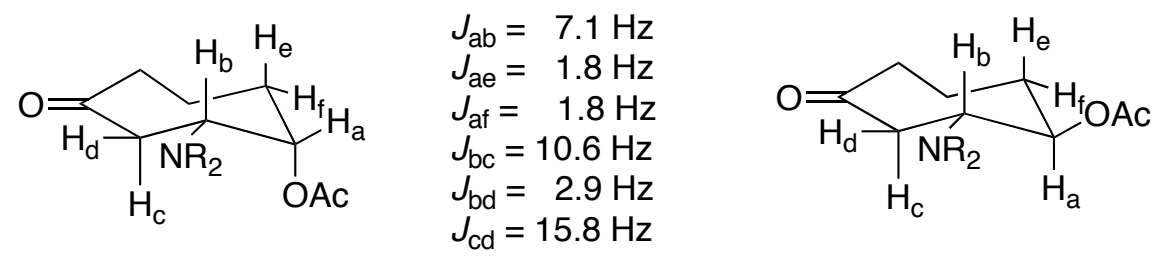

$J_{\mathrm{ab}}=9.8 \mathrm{~Hz}$
$J_{\mathrm{ae}}=9.8 \mathrm{~Hz}$
$J_{\mathrm{af}}=3.7 \mathrm{~Hz}$
$J_{\mathrm{bc}}=11.5 \mathrm{~Hz}$
$J_{\mathrm{bd}}=2.4 \mathrm{~Hz}$
$J_{\mathrm{cd}}=13.1 \mathrm{~Hz}$

anti Acetic acid 2-indazol-1-yl-4-oxo-cycloheptyl ester (20)(table 3, entry 6). ${ }^{\mathbf{1 3}}$ Isolated $30 \mathrm{mg}(34 \%)$ as a clear, colourless oil after column chromatography (50\% EtOAc/Hex). TLC $R_{\mathrm{f}} 0.23\left(50 \%\right.$ EtOAc/Hexanes); ${ }^{1} \mathrm{H}$ $\operatorname{NMR}\left(\mathrm{CDCl}_{3}, 300 \mathrm{MHz}\right) 7.94(\mathrm{~s}, 1 \mathrm{H}), 7.70(\mathrm{~d}, 1 \mathrm{H}, J=8.8 \mathrm{~Hz}), 7.63(\mathrm{dt}, 1 \mathrm{H}, J=8.4$ and $1.0 \mathrm{~Hz}), 7.32-7.26(\mathrm{~m}$, $1 \mathrm{H}), 7.08(\mathrm{ddd}, 1 \mathrm{H}, J=8.2,6.7$ and $0.6 \mathrm{~Hz}), 5.53(\mathrm{td}, 1 \mathrm{H}, J=9.8$ and $3.7 \mathrm{~Hz}), 4.61(\mathrm{ddd}, 1 \mathrm{H}, J=11.5,9,2$ and $2.4 \mathrm{~Hz}), 3.68(\mathrm{dd}, 1 \mathrm{H}, J=13.1$ and $11.7 \mathrm{~Hz}), 2.87(\mathrm{dd}, 1 \mathrm{H}, J=13.2$ and $2.5 \mathrm{~Hz}), 2.75(\mathrm{dtd}, 1 \mathrm{H}, J=17.3,4.7$ and $0.7 \mathrm{~Hz}), 2.58-2.47(\mathrm{~m}, 1 \mathrm{H}), 2.33-2.24(\mathrm{~m}, 1 \mathrm{H}), 2.11-1.98(\mathrm{~m}, 2 \mathrm{H}), 1.77(\mathrm{~s}, 3 \mathrm{H}), 1.69-1.55(\mathrm{~m}, 1 \mathrm{H}) ;{ }^{13} \mathrm{C}$ NMR (100 MHz) 211.8, 169.3, 126.5, 122.9, 122.1, 121.2, 117.6, 76.4, 63.0, 45.4, 43.4, 31.2, 20.7, 18.5; IR (film): 2943, 1736, 1704, 1376, 1233, $1032 \mathrm{~cm}^{-1}$; HRMS (EI): Exact mass calcd for $\mathrm{C}_{16} \mathrm{H}_{18} \mathrm{~N}_{2} \mathrm{O}_{3}[\mathrm{M}]^{+}$: 286.1317. Found: 286.1330.

(13) The relative stereochemistry of the O-acetoxy and indazol-1-yl substituents was ascertained by analysis of the ${ }^{1} \mathrm{H}$ NMR spectroscopy vicinal coupling constants. The syn isomer is indicated by $\mathrm{H}_{\mathrm{a}}$, which is a dt $(J=7.1$ and $1.8 \mathrm{~Hz})$ at $5.43 \mathrm{ppm}$. Conversely, the anti isomer is indicated by $\mathrm{H}_{\mathrm{a}}$, which is a td $(J=9.8$ and $3.7 \mathrm{~Hz})$ at $5.53 \mathrm{ppm}$. 


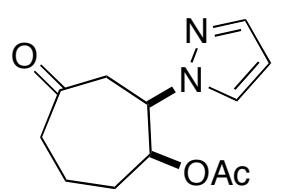

1.1

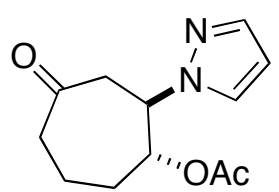

1

syn Acetic acid 4-oxo-2-pyrazol-1-yl-cycloheptyl ester (21)(table 3, entry 7). ${ }^{\mathbf{1 4}}$ Isolated $46 \mathrm{mg}(46 \%)$ as a clear, colourless oil after column chromatography $\left(2 \% \mathrm{MeOH} / \mathrm{CH}_{2} \mathrm{Cl}_{2}\right.$, then $\left.50 \% \mathrm{EtOAc} / \mathrm{Hexanes}\right)$. TLC $R_{\mathrm{f}}$ 0.28 (50\% EtOAc/Hexanes); ${ }^{1} \mathrm{H}$ NMR $\left(\mathrm{CDCl}_{3}, 400 \mathrm{MHz}\right) 7.49$ (br s, 1H), 7.37 (br s, 1H), 6.26 (br s, $\left.1 \mathrm{H}\right), 5.31$ (br d, $1 \mathrm{H}, J=7.1 \mathrm{~Hz}), 4.82-4.79(\mathrm{~m}, 1 \mathrm{H}), 3.47(\mathrm{dd}, 1 \mathrm{H}, J=15.7$ and $10.4 \mathrm{~Hz}), 2.86(\mathrm{dd}, 1 \mathrm{H}, J=15.8$ and 2.6 $\mathrm{Hz}$ ), 2.80 (ddd, $1 \mathrm{H}, J=11.3,8.6$ and $3.4 \mathrm{~Hz}), 2.57(\mathrm{ddd}, 1 \mathrm{H}, J=16.4,10.0$ and $3.0 \mathrm{~Hz}), 2.20-2.12(\mathrm{~m}, 1 \mathrm{H})$, $2.06(\mathrm{~s}, 3 \mathrm{H}), 1.96-1.75(\mathrm{~m}, 3 \mathrm{H}) ;{ }^{13} \mathrm{C}$ NMR $(100 \mathrm{MHz}) 208.9,169.5,139.2,128.1,106.1,74.8,59.8,43.7,43.6$, 31.1, 21.0, 18.4; IR (film): 3119, 3060, 2943, 2870, 1736, 1704, 1628, 1376, 1233, $1032 \mathrm{~cm}^{-1}$; HRMS (EI): Exact mass calcd for $\mathrm{C}_{12} \mathrm{H}_{16} \mathrm{~N}_{2} \mathrm{O}_{3}[\mathrm{M}]^{+}: 236.1161$. Found: 236.1141 .

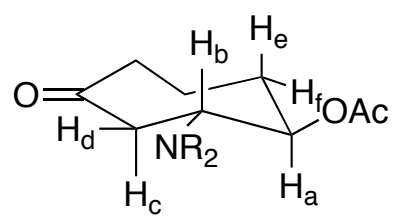

$$
\begin{aligned}
& J_{\mathrm{ab}}=9.2 \mathrm{~Hz} \\
& J_{\mathrm{ae}}=9.9 \mathrm{~Hz} \\
& J_{\mathrm{af}}=3.7 \mathrm{~Hz} \\
& J_{\mathrm{bc}}=11.4 \mathrm{~Hz} \\
& J_{\mathrm{bd}}=2.5 \mathrm{~Hz} \\
& J_{\mathrm{cd}}=13.3 \mathrm{~Hz}
\end{aligned}
$$

anti Acetic acid 4-oxo-2-pyrazol-1-yl-cycloheptyl ester (21)(table 3, entry 7). ${ }^{\mathbf{1 4}}$ Isolated $41 \mathrm{mg}(41 \%)$ as a clear, colourless oil after column chromatography $\left(2 \% \mathrm{MeOH} / \mathrm{CH}_{2} \mathrm{Cl}_{2}\right.$, then $\left.50 \% \mathrm{EtOAc} / \mathrm{Hex}\right)$. TLC $R_{\mathrm{f}} 0.25$ (50\% EtOAc/Hexanes); ${ }^{1} \mathrm{H}$ NMR $\left(\mathrm{CDCl}_{3}, 400 \mathrm{MHz}\right) 7.51$ (br d, $\left.1 \mathrm{H}, J=1.5 \mathrm{~Hz}\right), 7.37$ (br d, $\left.1 \mathrm{H}, 1.5 \mathrm{~Hz}\right), 6.20$ (t, $1 \mathrm{H}, J=2.1 \mathrm{~Hz}$ ), 5.33 (ddd, $1 \mathrm{H}, J=9.9,9.2$ and $3.7 \mathrm{~Hz}$ ), 4.31 (ddd, $1 \mathrm{H}, J=11.4,9.0,2.5 \mathrm{~Hz}$ ), 3.47 (dd, $1 \mathrm{H}, J$ $=13.3$ and $11.4 \mathrm{~Hz}), 2.82(\mathrm{dd}, 1 \mathrm{H}, J=13.4$ and $2.5 \mathrm{~Hz}), 2.69(\mathrm{dtd}, 1 \mathrm{H}, J=17.4,4.9$ and $0.9 \mathrm{~Hz}), 2.48(\mathrm{ddd}, 1 \mathrm{H}$, $J=17.4,10.6,5.3 \mathrm{~Hz}), 2.23-2.17(\mathrm{~m}, 1 \mathrm{H}), 2.07-1.90(\mathrm{~m}, 2 \mathrm{H}), 1.85(\mathrm{~s}, 3 \mathrm{H}), 1.60$ (dddd, $1 \mathrm{H}, J=14.3,10.2,10.1$ and $2.1 \mathrm{~Hz}) ;{ }^{13} \mathrm{C}$ NMR $(100 \mathrm{MHz}) 209.6,169.2,139.9,129.2,105.3,76.1,61.3,45.1,43.3,31.3,20.7,18.5 ; \mathrm{IR}$ (film): 3121, 3061, 2940, 1738, 1702, 1376, 1233, $1028 \mathrm{~cm}^{-1}$; HRMS (EI): Exact mass calcd for $\mathrm{C}_{12} \mathrm{H}_{16} \mathrm{~N}_{2} \mathrm{O}_{3}$ $[\mathrm{M}]^{+}:$236.1161. Found: 236.1183.<smiles>[2H]c1nc2ccccc2[nH]1</smiles>

1,2-Dideuterobenzimidazole (22)(equation 3). Oxalyl chloride (0.048 g, $0.38 \mathrm{mmol})$ was added slowly to a 25 $\mathrm{mL}$ round bottom flask containing $8 \mathrm{~mL} \mathrm{D}_{2} \mathrm{O}$ under magnetic stirring. The solution was refluxed for $1 \mathrm{~h}$ and then cooled to room temperature. Benzimidazole $(0.443 \mathrm{~g}, 3.75 \mathrm{mmol})$ was then added to the solution, and the heterogenous mixture was then refluxed for two hours to give a clear homogeneous solution. Upon cooling to room temperature over 20 minutes, precipitation of white crystals was observed. The mixture was filtered in a glass frit and the crystals were washed with diethyl ether, followed by drying under reduced pressure to give the product as white needles $(0.430 \mathrm{~g}, 95 \%) .{ }^{1} \mathrm{H} \mathrm{NMR}\left(\mathrm{CDCl}_{3}, 400 \mathrm{MHz}\right) 7.69(\mathrm{dd}, 2 \mathrm{H}, J=6.1$ and $3.2 \mathrm{~Hz}), 7.31$

(14) The relative stereochemistry of the $O$-acetoxy pyrazol-1-yl substituents was ascertained by analysis of the ${ }^{1} \mathrm{H}$ NMR spectroscopy vicinal coupling constants for the anti isomer. Due to broad signals, the relevant coupling constants could not be extracted for the remaining isomer. It was thus assigned as the syn isomer by process of elimination as well as by analogy with compound 20. 
(dd, 2H, $J=6.1$ and $3.2 \mathrm{~Hz}) ;{ }^{13} \mathrm{C}$ NMR (100 MHz) 137.3, 123.1, 115.5; IR (film): 3136, 3073, 3045, 3026, 2981, 2960, 2824, 2786, 2725, 2678, 2606, 1590, 1565, 1553, 1439, $913 \mathrm{~cm}^{-1}$; HRMS (EI): Exact mass calcd for $\mathrm{C}_{7} \mathrm{H}_{4} \mathrm{D}_{2} \mathrm{~N}_{2}[\mathrm{M}]^{+}: 120.0657$. Found: 120.0677 .

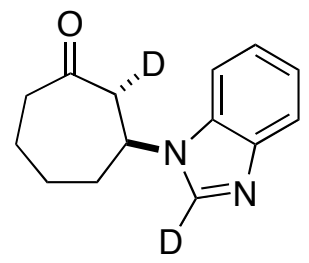

Procedure for Deuterium Labelling Experiment for Determination of Stereochemistry (3i)(equation 3). ${ }^{15}$ A borosilicate tube was charged with a stir bar, cyclohept-2-enone $(0.025 \mathrm{~g}, 0.23 \mathrm{mmol})$ and 1,2dideuterobenzimidazole (22) $(0.080 \mathrm{~g}, 0.68 \mathrm{mmol})$. A volume of $4.0 \mathrm{~mL}$ dichloromethane and $0.5 \mathrm{~mL}$ of $\mathrm{CD}_{3} \mathrm{CN}$ was added to the mixture. The tube was capped with a septum and was purged with a nitrogen balloon and an outlet for 5 minutes while stirring. The tube was then placed between two Luzchem exposure panels each equipped with four $8 \mathrm{~W}$ UV-A bulbs for 16 hours while stirring. The reaction was monitored by TLC and was found to have $R_{\mathrm{f}} 0.56$ on silica gel $\left(10 \% \mathrm{MeO} / \mathrm{CH}_{2} \mathrm{Cl}_{2}\right)$. The crude mixture was then transferred to a round bottom flask and the solvent was evaporated under reduced pressure. The resulting oil was purified by column chromatography using $4 \% \mathrm{MeOH} / \mathrm{CH}_{2} \mathrm{Cl}_{2}$. The product was a clear, colorless oil (39 mg, $75 \%$ ); ${ }^{1} \mathrm{H} \mathrm{NMR}$ $\left(\mathrm{CDCl}_{3}, 400 \mathrm{MHz}\right) \delta$ 7.83-7.79 (m, 1H), 7.44-7.40 (m, 1H), 7.34-7.26 (m, 2H), $4.53(\mathrm{ddd}, J=11.5,11.5$ and 3.0 $\mathrm{Hz}, 1 \mathrm{H}), 3.27(\mathrm{~d}, J=11.5 \mathrm{~Hz}, 1 \mathrm{H}), 2.74-2.32(\mathrm{~m}, 3 \mathrm{H}), 2.25-2.02(\mathrm{~m}, 3 \mathrm{H}), 1.86-1.73(\mathrm{~m}, 1 \mathrm{H}), 1.63-1.50(\mathrm{~m}$, $1 \mathrm{H}) ;{ }^{13} \mathrm{C}$ NMR (100 MHz) 209.8, 123.6, 123.0, 121.1, 110.4, 54.0 50.4, 44.4, 38.0, 27.4, 24.2; IR (film): 2936, 2872, 1699, 1489, 1458, 1283, 1221, 1206, 1082, $744 \mathrm{~cm}^{-1}$; HRMS (EI): Exact mass calcd for $\mathrm{C}_{14} \mathrm{H}_{14} \mathrm{D}_{2} \mathrm{~N}_{2} \mathrm{O}$ $[\mathrm{M}]^{+}: 230.1388$. Found: 230.1401 .

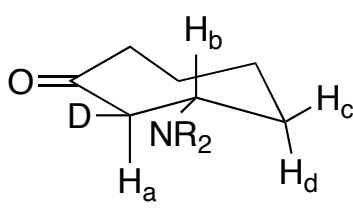

$$
\begin{aligned}
& J_{a b}=11.5 \mathrm{~Hz} \\
& J_{b c}=3.0 \mathrm{~Hz} \\
& J_{b d}=11.5 \mathrm{~Hz}
\end{aligned}
$$

Procedure for Low Temperature Generation/Trapping of (E)-Cyclooct-2-enone (equation 4). ${ }^{16,17}$ A borosilicate tube was charged with a stir bar, $(Z)$-cyclooct-2-enone $(0.025 \mathrm{~g}, 0.20 \mathrm{mmol})$ and $3.0 \mathrm{~mL}$ of a $8: 1$ $\mathrm{CH}_{2} \mathrm{Cl}_{2} / \mathrm{MeCN}$. The volume was marked on the tube and an excess of $1-2 \mathrm{~mL}$ of $\mathrm{CH}_{2} \mathrm{Cl}_{2}$ was then added. The tube was capped with a septum and was purged with stirring using a nitrogen balloon and an outlet for at least 5 min or until the solvent evaporated to the volume marked. The solution was cooled to $-75{ }^{\circ} \mathrm{C}$ and was allowed to sit between two exposure panels each equipped with four $8 \mathrm{~W} \mathrm{UV-A}$ bulbs for $1 \mathrm{hr}$, maintaining cooling. ${ }^{18}$

(15) Despite multiple attempts, it was not possible to obtain completely isotopically pure $\mathbf{3 i}$ at the C-2 position. This is attributed to imperfect deuteration of $\mathbf{2 2}$ and a potential kinetic isotope effect during the reaction.

(16) For examples of low temperature generation/trapping experiments with (E)-cycloalkenones, see: (a) Corey, E. J.; Tada, M.; LaMahieu, R.; Libit, L. J. Am. Chem. Soc. 1965, 87, 2051. (b) Noyori, R.; Watanabe, A.; Katô, M. Tetrahedron Lett. 1968, 52, 5443. (c) Katô, M.; Noyori, R. Bull. Chem. Soc. Jpn. 1974, 47, 1460.

(17) For examples of low temperature generation/trapping experiments with (E)-cycloalkenes, see: (a) Inoue, Y.; Ueoka, T.; Kuroda, T.; Hakushi, T. J. Chem. Soc., Chem. Commun. 1981, 1031. (b) Inoue, Y.; Ueoka, T.; Kuroda, T.; Hakushi, T. J. Chem. Soc., Perkin Trans. 2 1983, 983. (c) Steinmetz, M. G.; Seguin, K. J.; Udayakumar, B. S.; Behnke, J. S. J. Am. Chem. Soc. 1990, 112, 6601.

(18) The sample was immersed in a $95 \%$ ethanol bath contained in a $5 \mathrm{~L}$ quartz Dewar and cooled to $-75^{\circ} \mathrm{C}$ by an FTS Systems FC100 immersion cooler. 
Irradiation was stopped, and a heterogeneous mixture of benzimidazole $(0.095 \mathrm{~g}, 0.80 \mathrm{mmol})$ in $1.5 \mathrm{~mL} 8: 1$ $\mathrm{CH}_{2} \mathrm{Cl}_{2} / \mathrm{MeCN}$, pre-cooled to $-75{ }^{\circ} \mathrm{C}$, was then added by syringe to the borosilicate tube. ${ }^{19}$ The reaction mixture was allowed to gradually warm to room temperature while stirring under $\mathrm{N}_{2}$ for 45 minutes. The formation of a new product was observed by TLC $\left(R_{\mathrm{f}} 0.69\right.$ in $\left.10 \% \mathrm{MeOH} / \mathrm{CH}_{2} \mathrm{Cl}_{2}\right)$. The solvent was evaporated under reduced pressure and the crude reaction mixture was completely dissolved in $d_{6}$-DMSO. ${ }^{1} \mathrm{H}$ NMR of the crude reaction mixture indicated the conversion of $18 \%$ of the starting material to 3-benzoimidazol1-yl-cyclooctanone, based on the integration of the resonance corresponding to the product's $\mathrm{C} 3$ methine proton at $4.83 \mathrm{ppm}$ relative to the resonance corresponding to the enone's $\mathrm{C} 3 \mathrm{sp}^{2}$ methine proton at $6.36 \mathrm{ppm}$.

Procedure for Low Temperature NMR experiments with $(E)$-Cyclooct-2-enone. To an NMR tube was added (Z)-Cyclooct-2-enone $((Z)-11)(6 \mathrm{mg}, 0.05 \mathrm{mmol})$, followed by $1.0 \mathrm{~mL}$ of an $8: 1 \mathrm{CD}_{2} \mathrm{Cl}_{2} / \mathrm{CD}_{3} \mathrm{CN}$ solution. The tube was capped with a septum and exposed to three quick freeze/pump/thaw cycles to remove molecular oxygen and was backfilled with nitrogen. The solution was cooled to $-75^{\circ} \mathrm{C}$ and was allowed to sit between two exposure panels each equipped with four $8 \mathrm{~W} \mathrm{UV-A}$ bulbs for $45 \mathrm{~min}$, maintaining cooling. ${ }^{18}$ The NMR tube was then stored in a bath of dry ice in acetone $\left(-78^{\circ} \mathrm{C}\right)$ for approx. 5 min until it could be transferred to a Bruker Avance500 NMR spectrometer, pre-cooled to $-20{ }^{\circ} \mathrm{C}$. An initial spectrum was taken, indicating a newly formed compound with alkene resonances at $6.46(\mathrm{~d}, J=18.0 \mathrm{~Hz}, 1 \mathrm{H})$ and $5.69(\mathrm{ddd}, J=18.0,10.9$ and 4.1 Hz, 1H) ppm. The tube was then ejected, and benzimidazole $(18 \mathrm{mg}, 0.15 \mathrm{mmol})$ was added. The tube was quickly inverted to allow mixing and was reintroduced into the NMR. Another spectrum was taken at $-20{ }^{\circ} \mathrm{C}$ which indicated that no reaction occurred at this temperature. The NMR was allowed to warm to $10{ }^{\circ} \mathrm{C}$, and spectra were taken every 5 minutes. After 20 minutes, the $(\boldsymbol{E}) \mathbf{- 1 1}$ had reacted efficiently to afford 12, as judged by the relative ratios of integration of the relevant peaks, while the $(Z)-11$ remained unreacted.

(19) Benzimidazole was pre-ground with a mortar and pestle to maximize its surface area. 


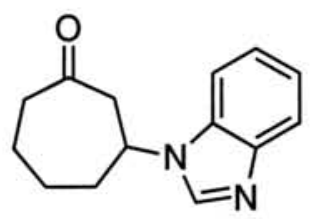

Table 2, Entry 1 3a
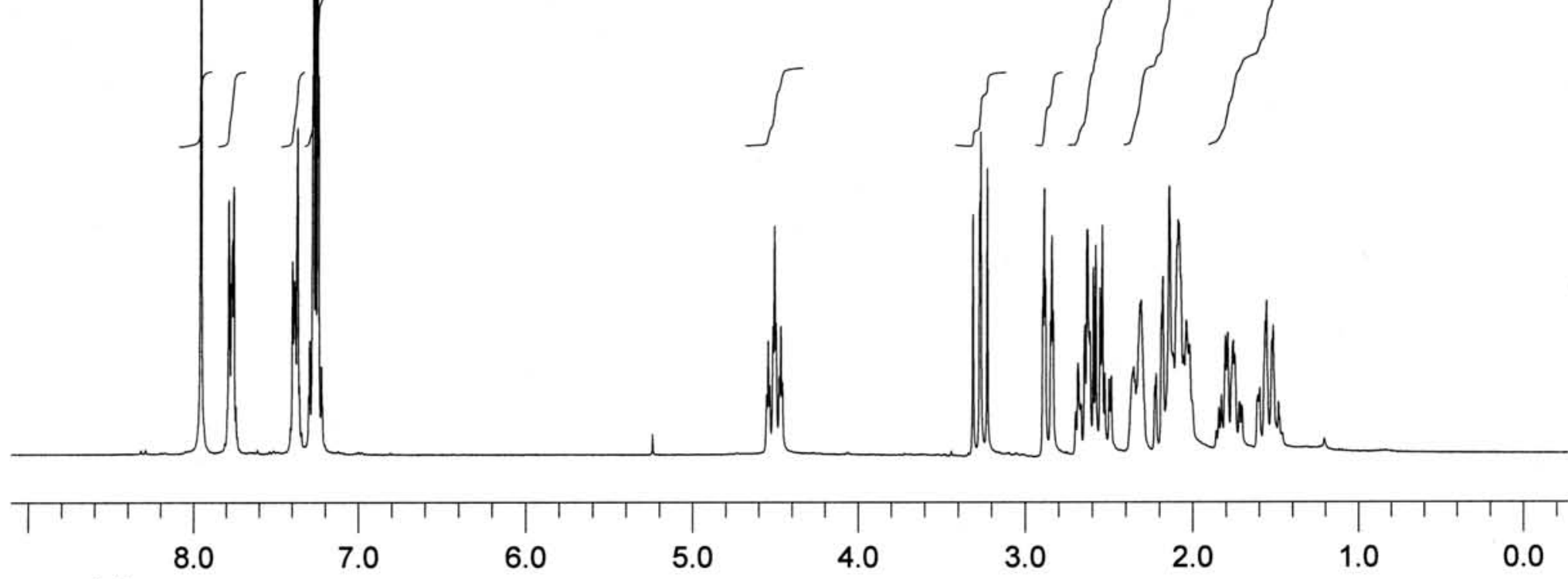
ppm (t1)

क्ष

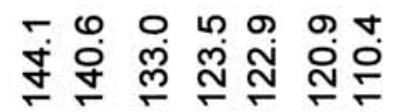

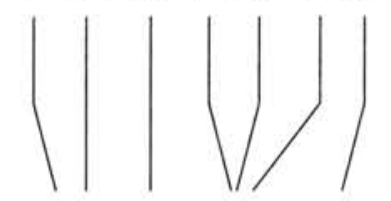

ભై

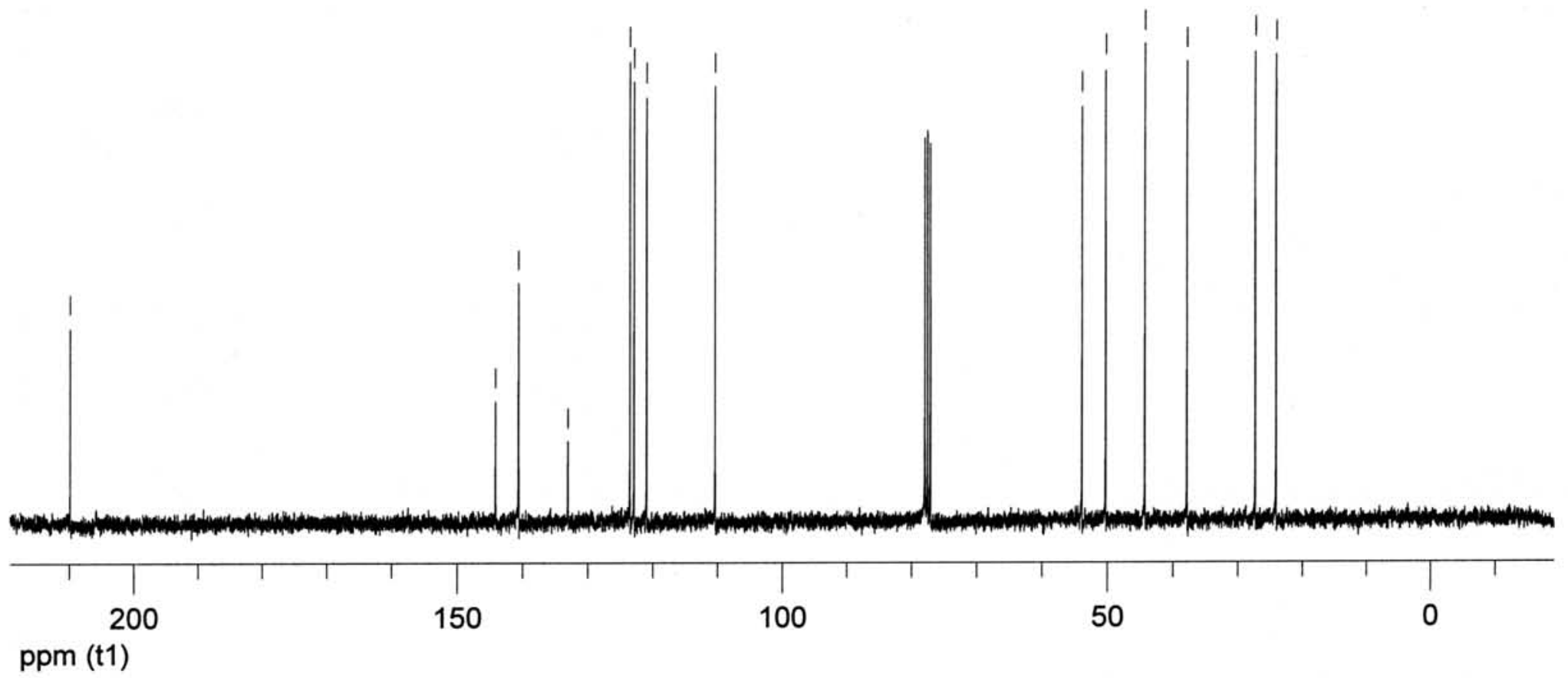




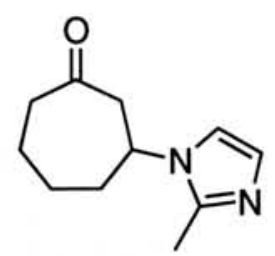

Table 2, Entry 2 3b

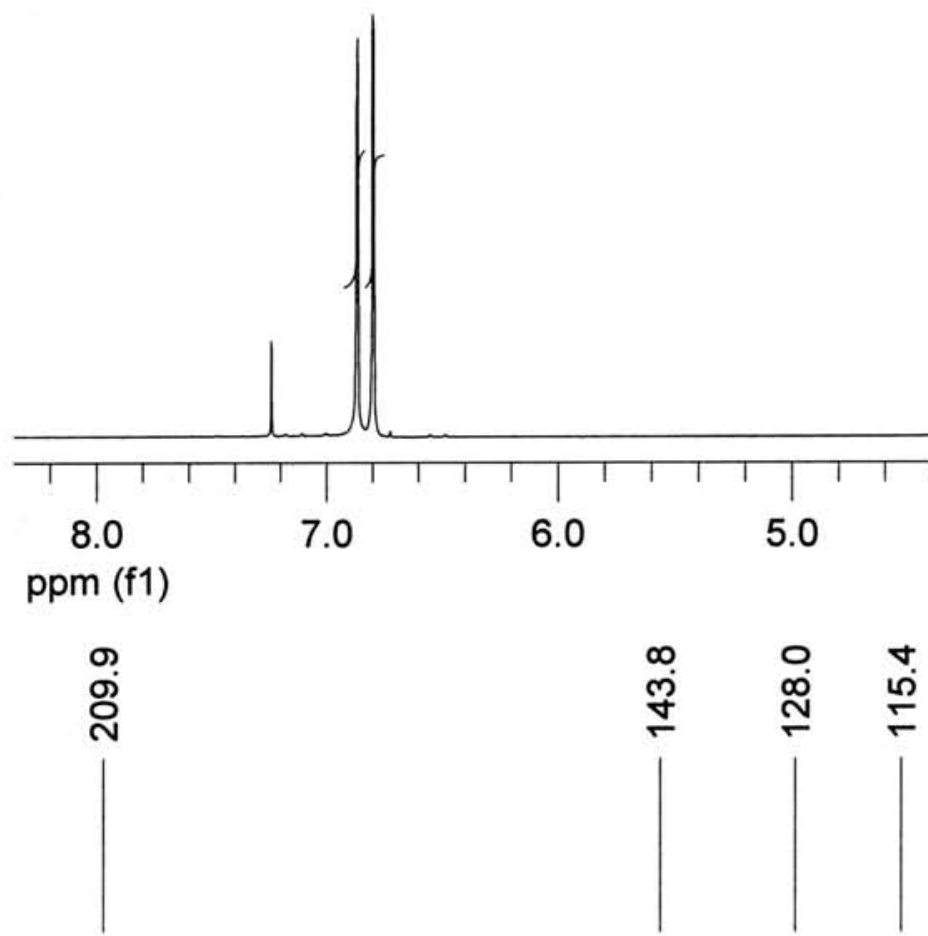

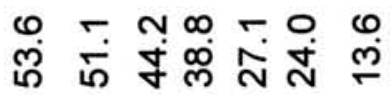
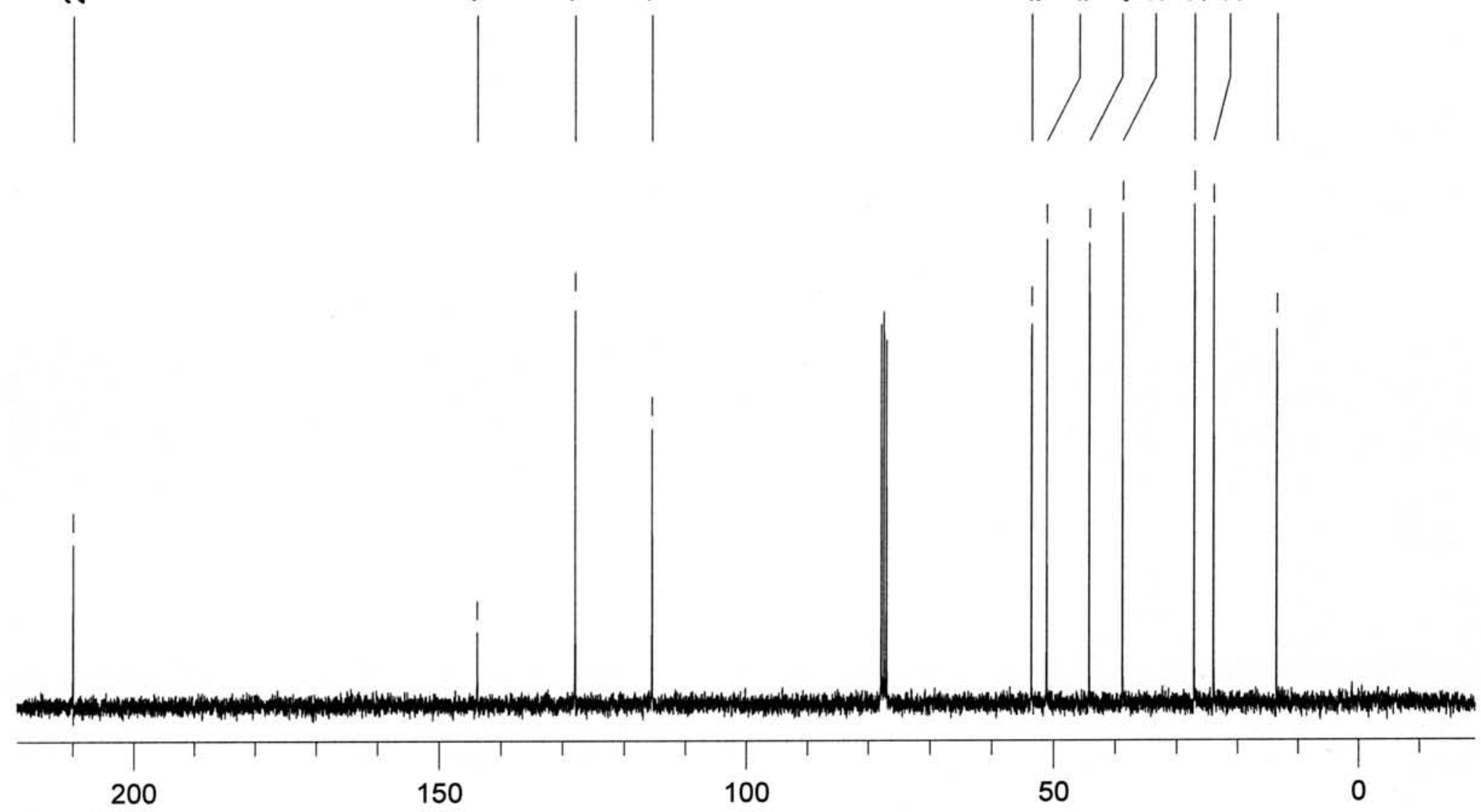

ppm (f1) 


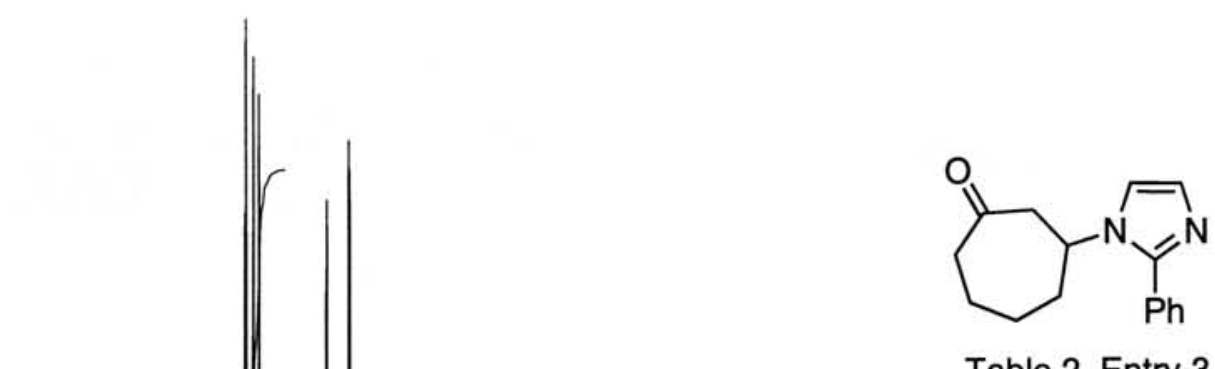

Table 2, Entry 3 3c

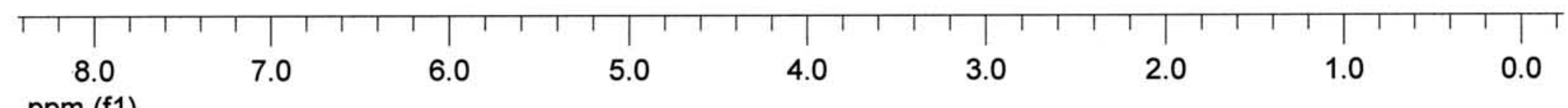
ppm (f1)

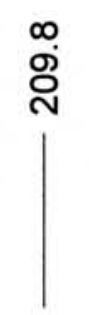

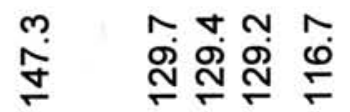

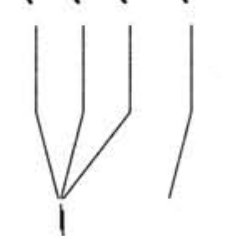

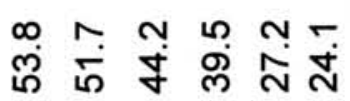

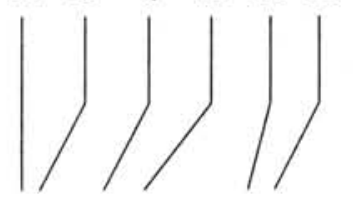

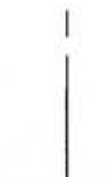

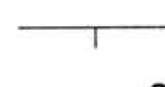

200

ppm (f1) 


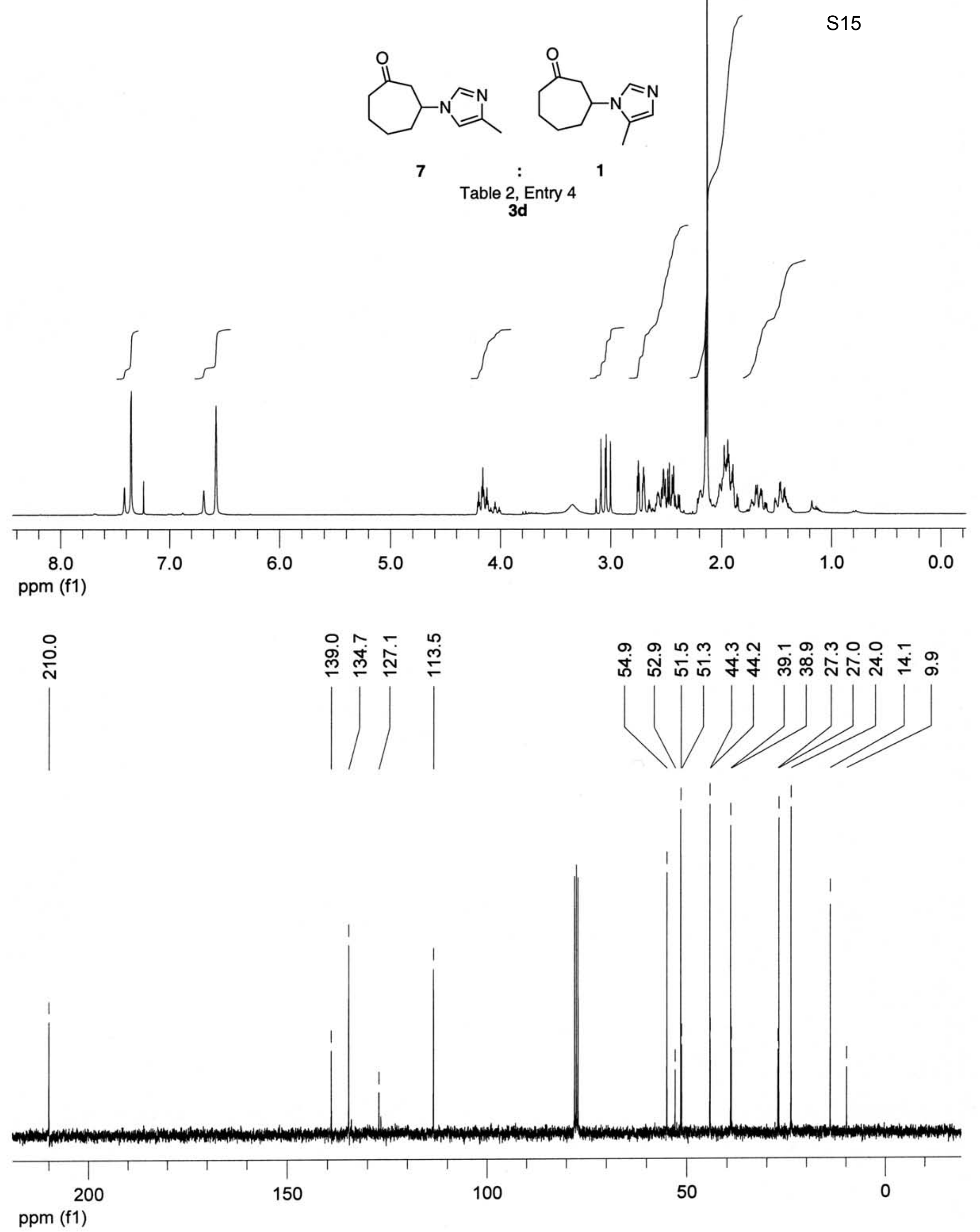



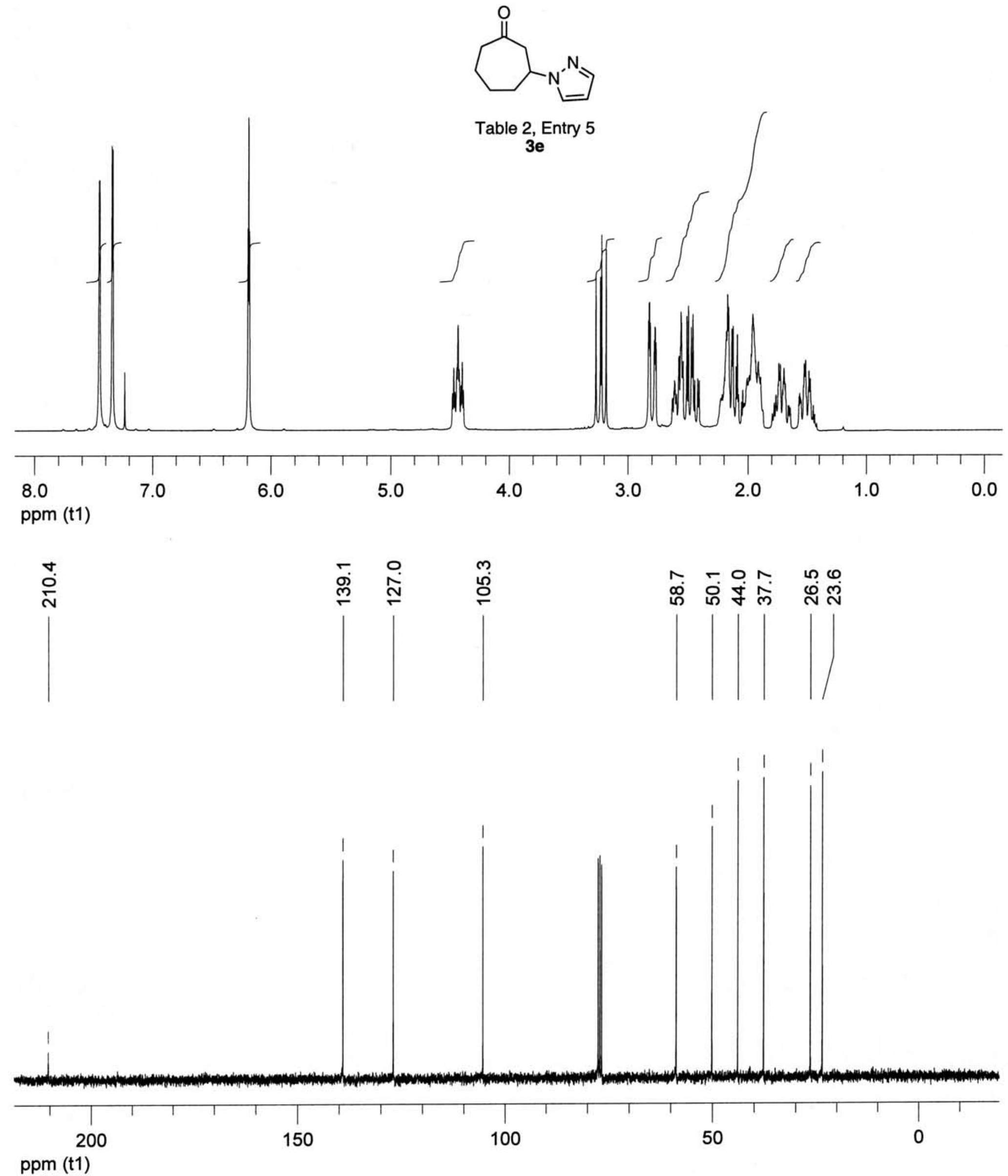


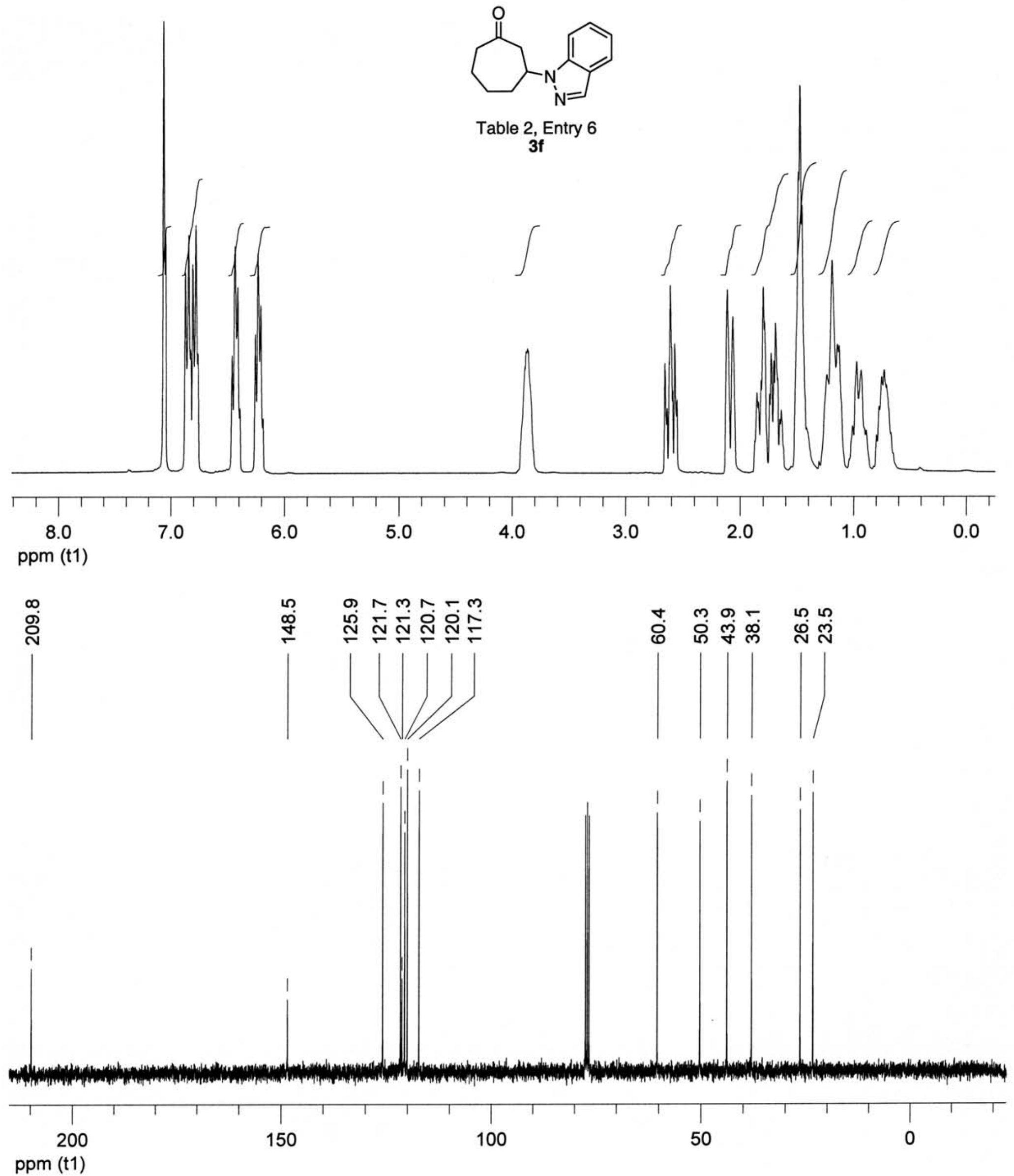



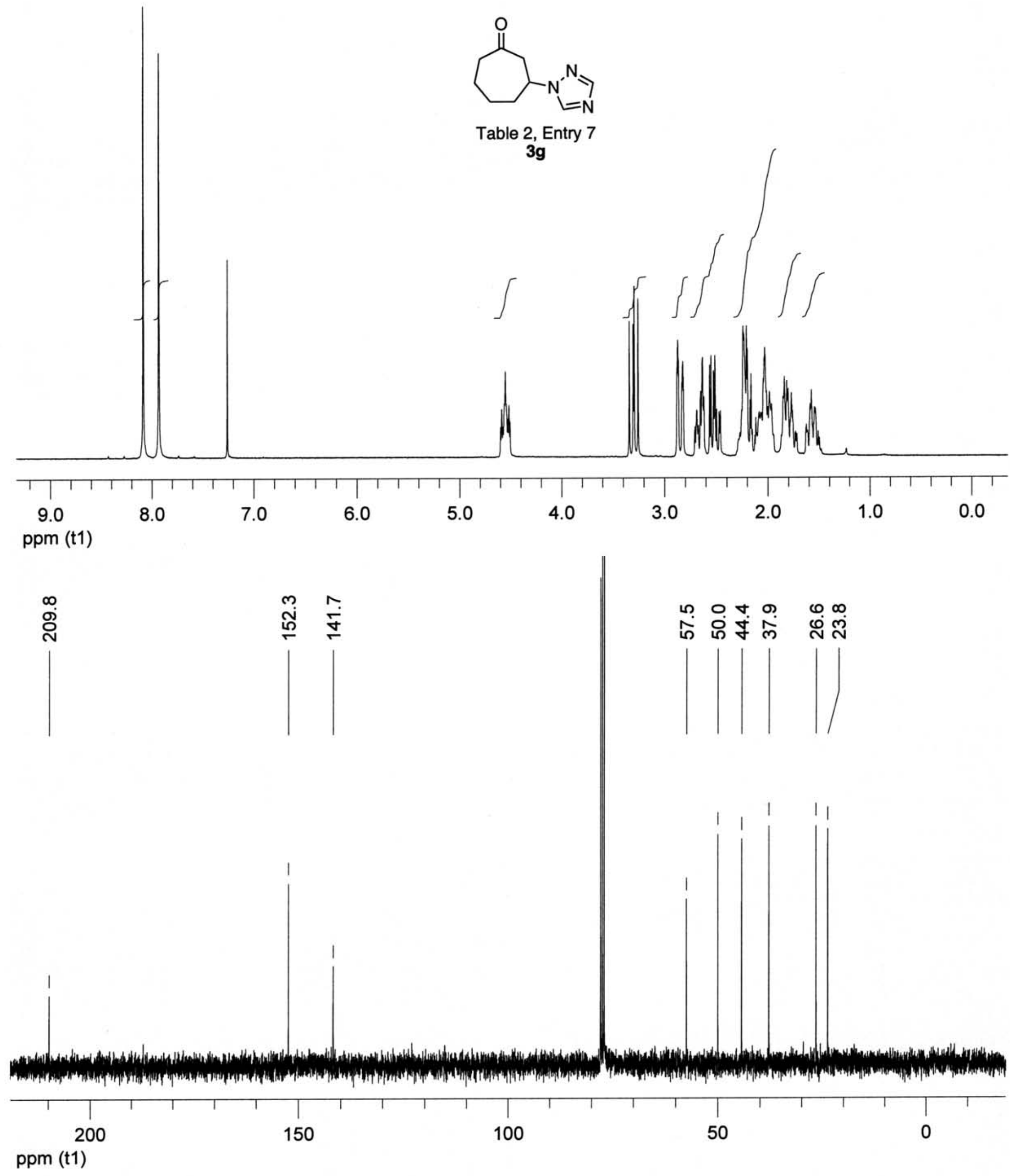


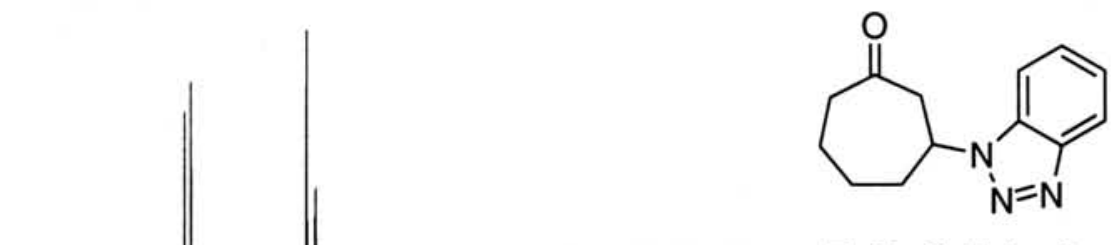

Table 2, Entry 8

$3 \mathrm{~h}$
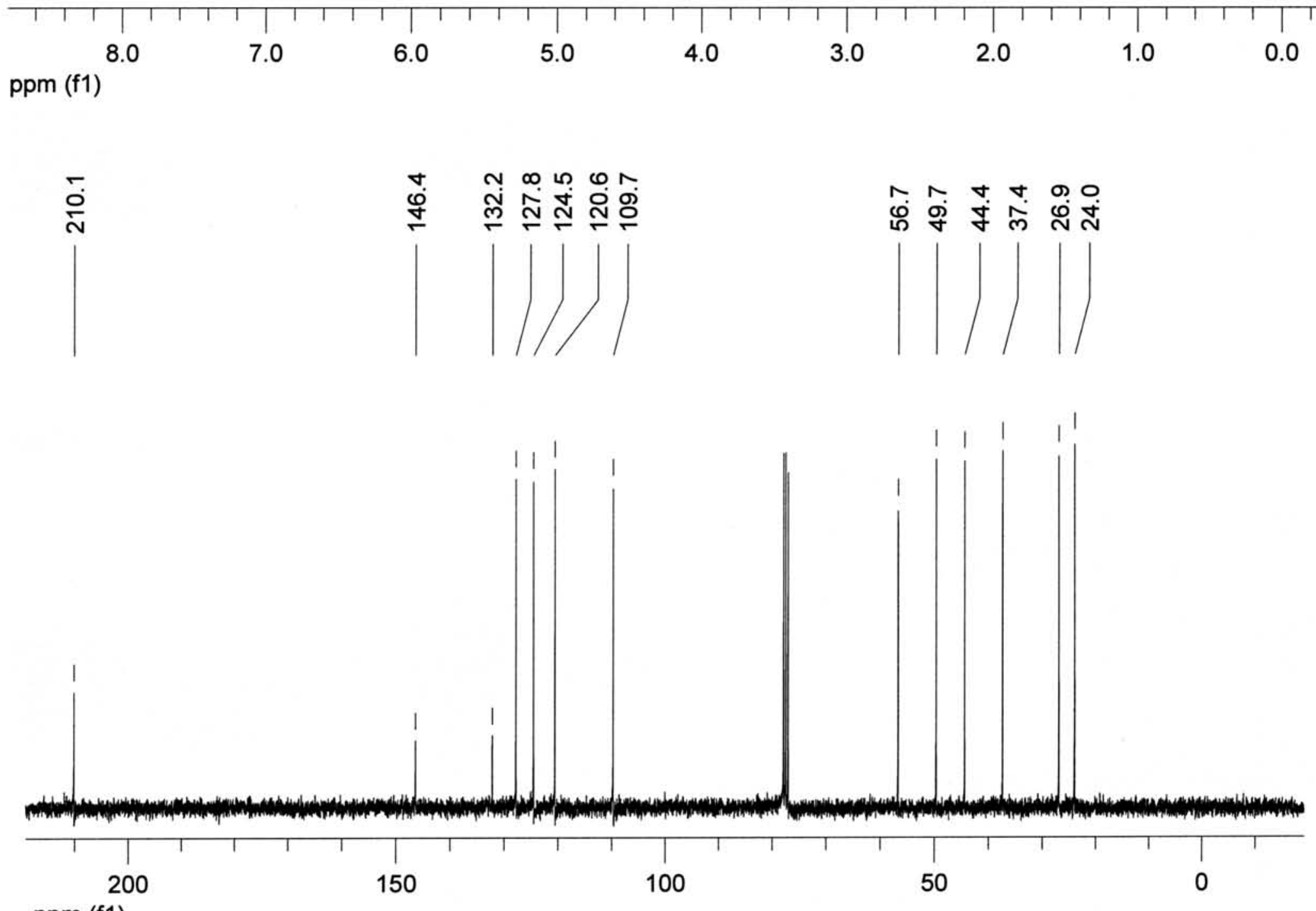

ppm (f1) 


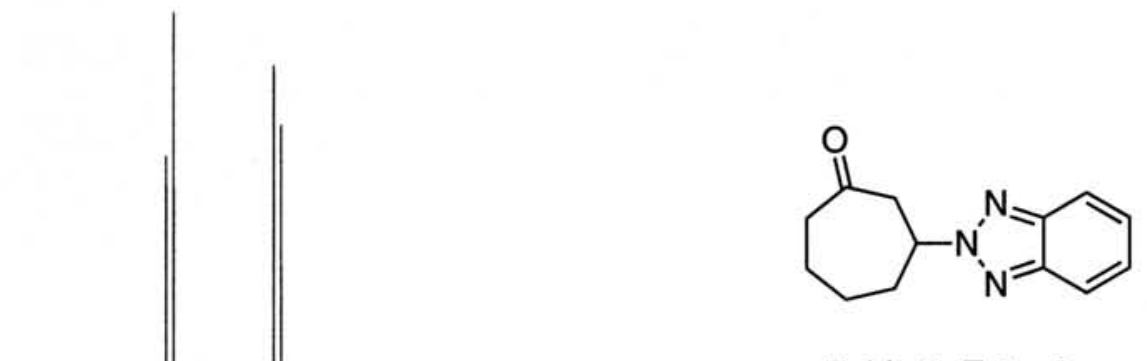

Table 2, Entry 8

3h
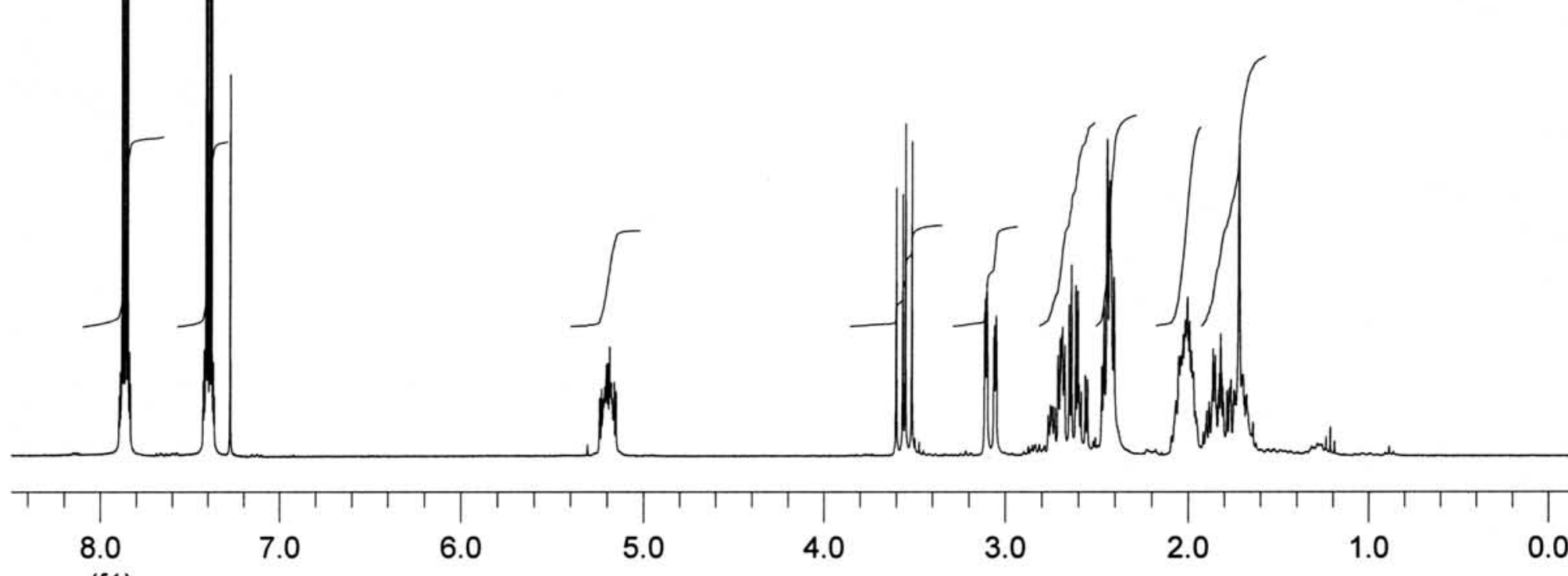

ppm (f1)

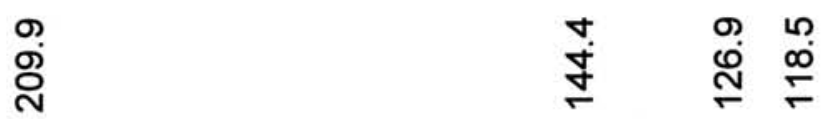

ก̋
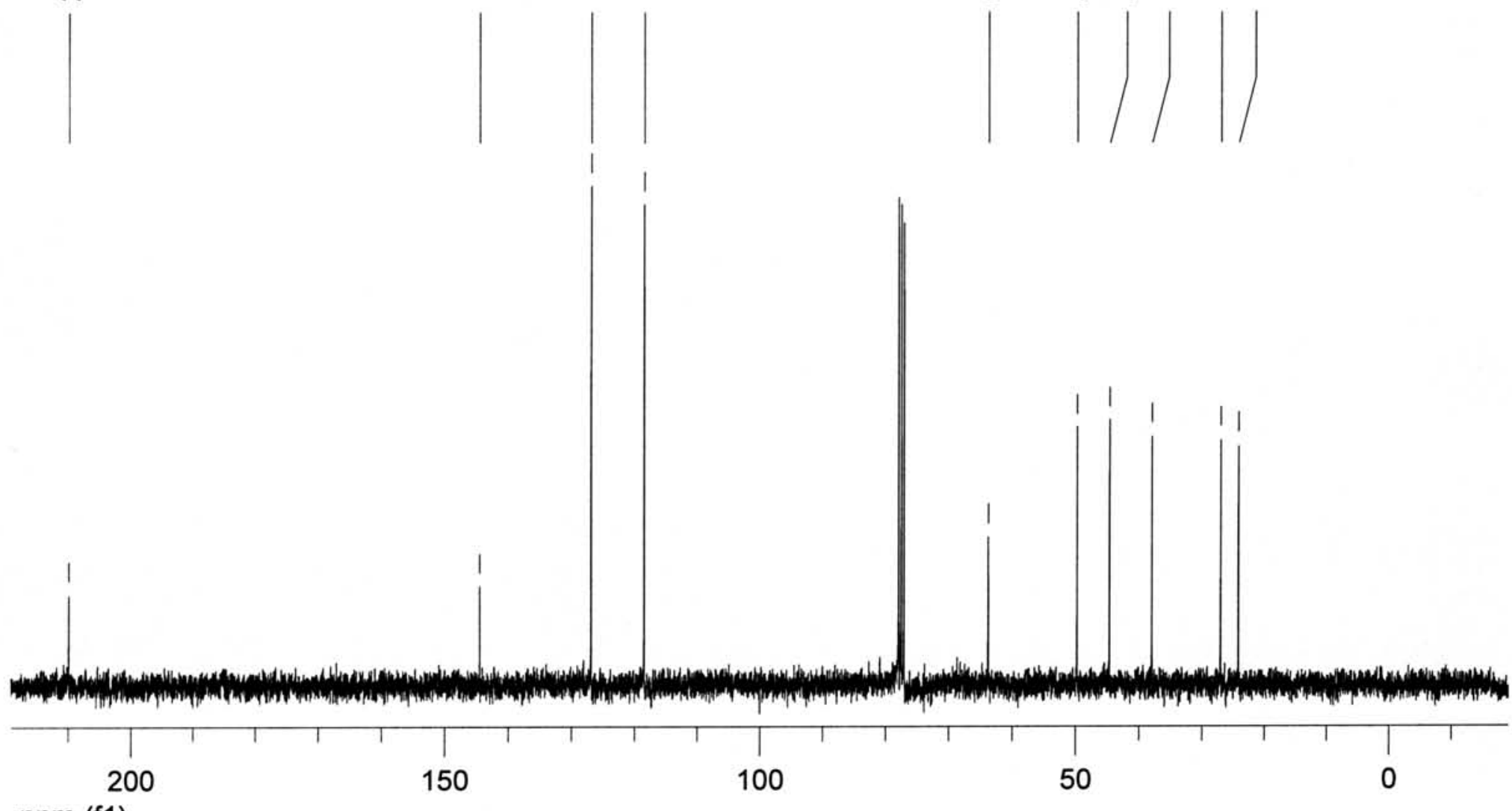

ppm (f1) 

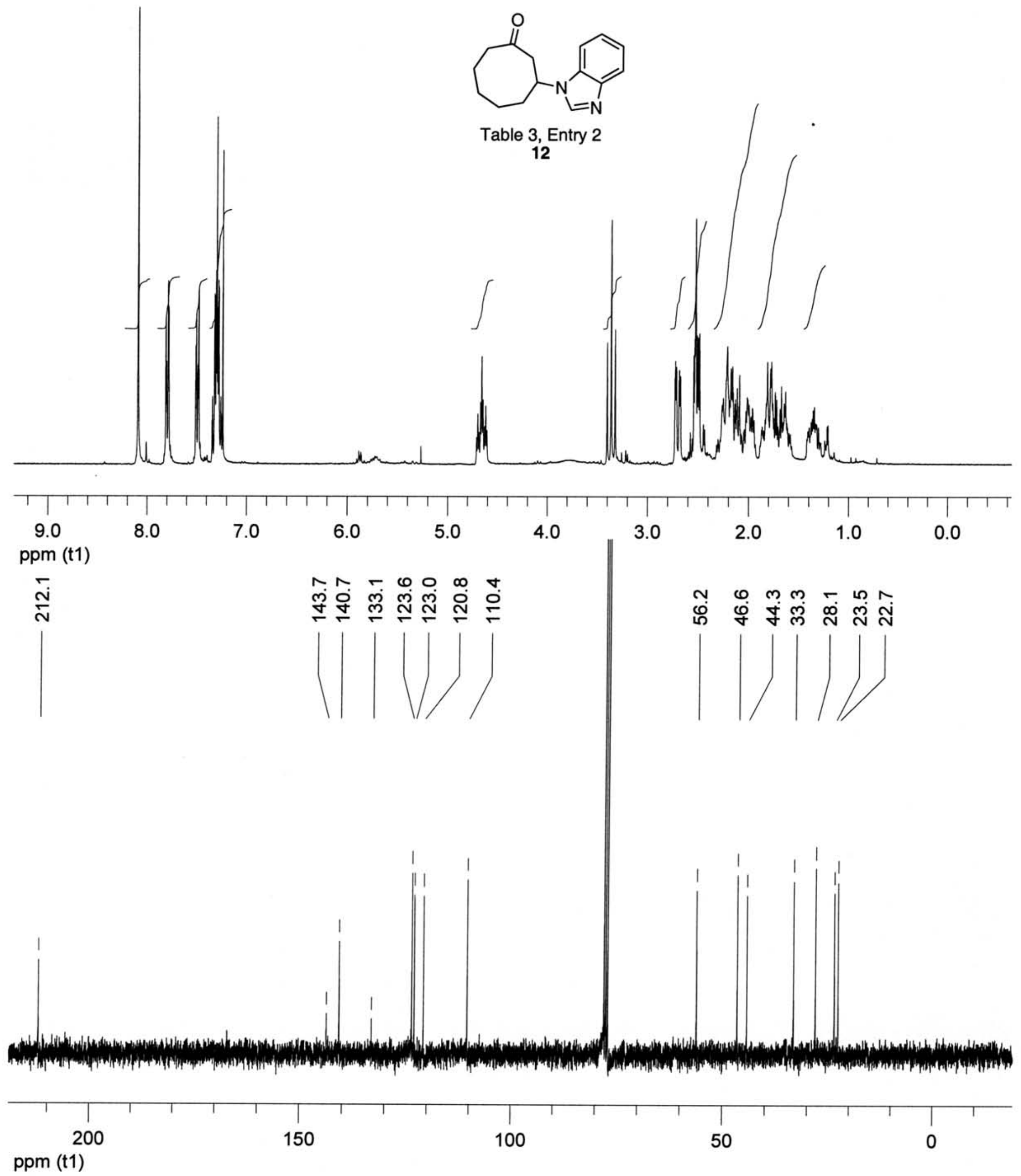


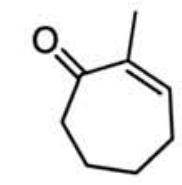

Table 3, Entry 3 13
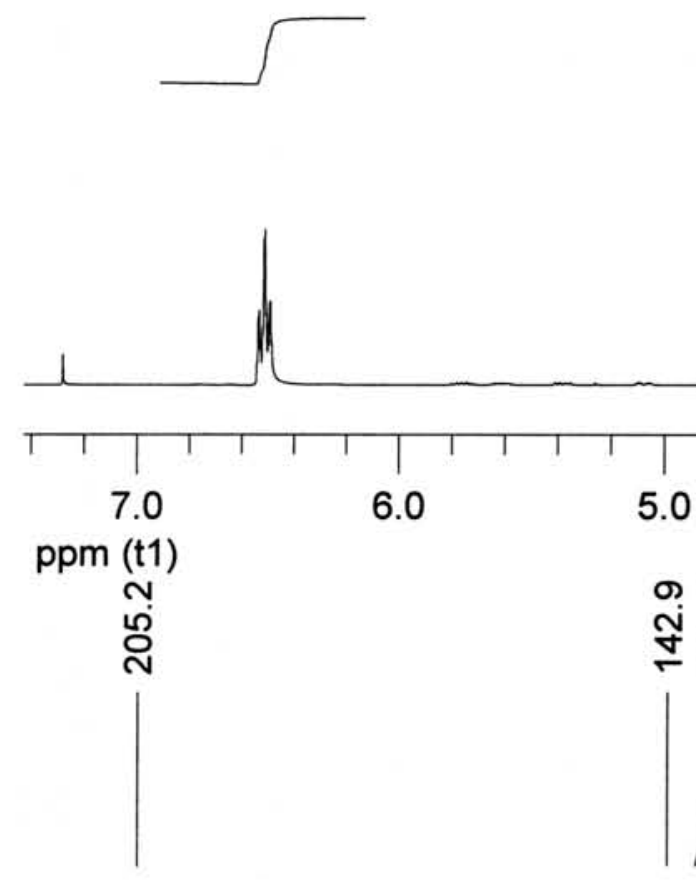

5.0

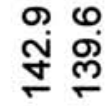

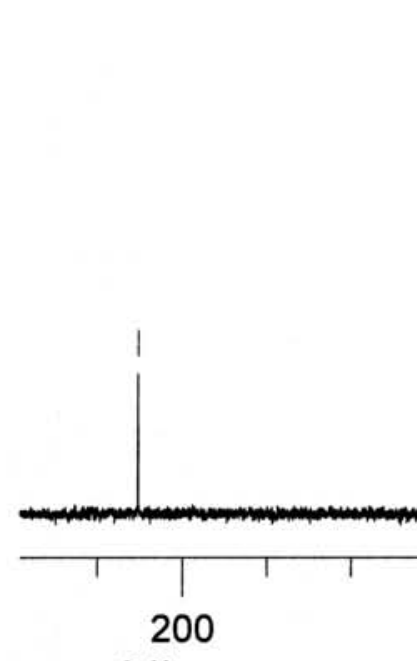

ppm (t1)
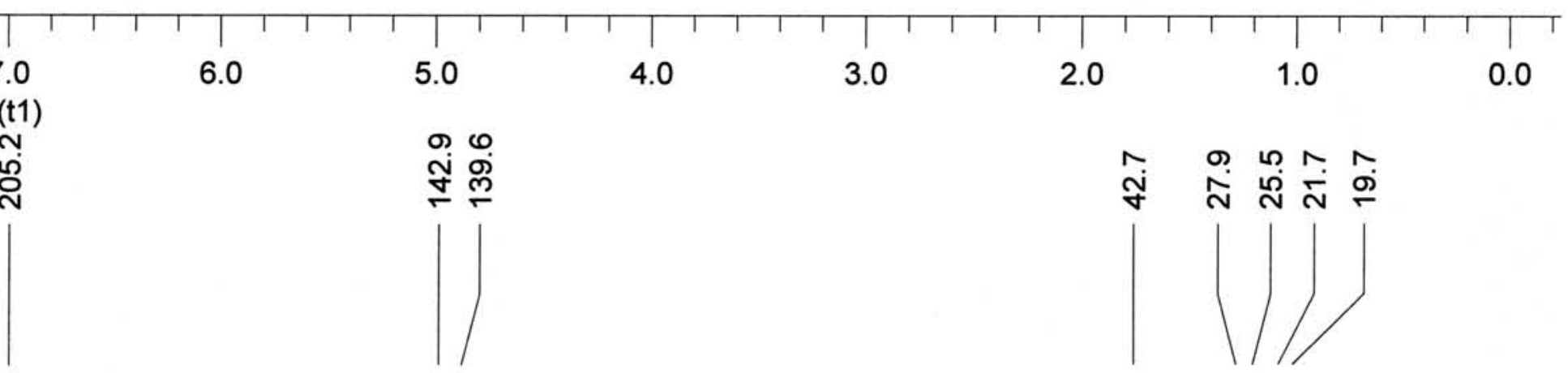

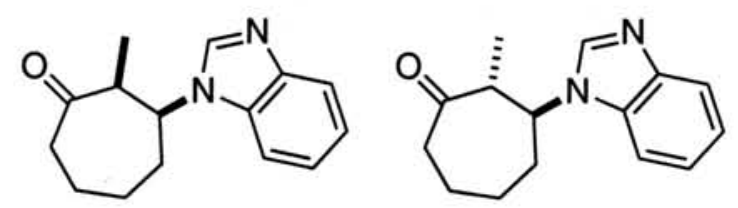

12

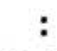

Table 3, Entry 3

\section{1}

14
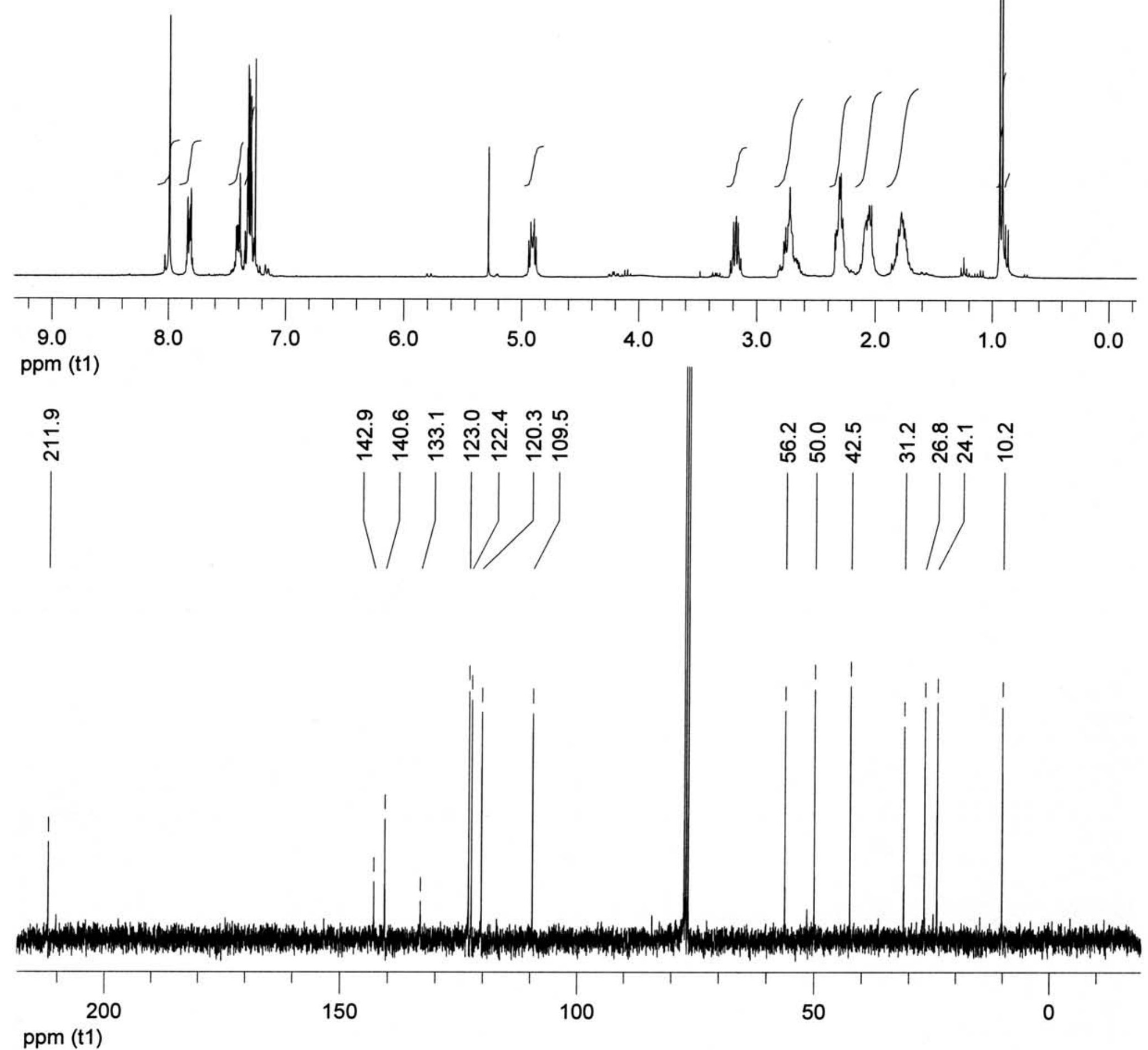


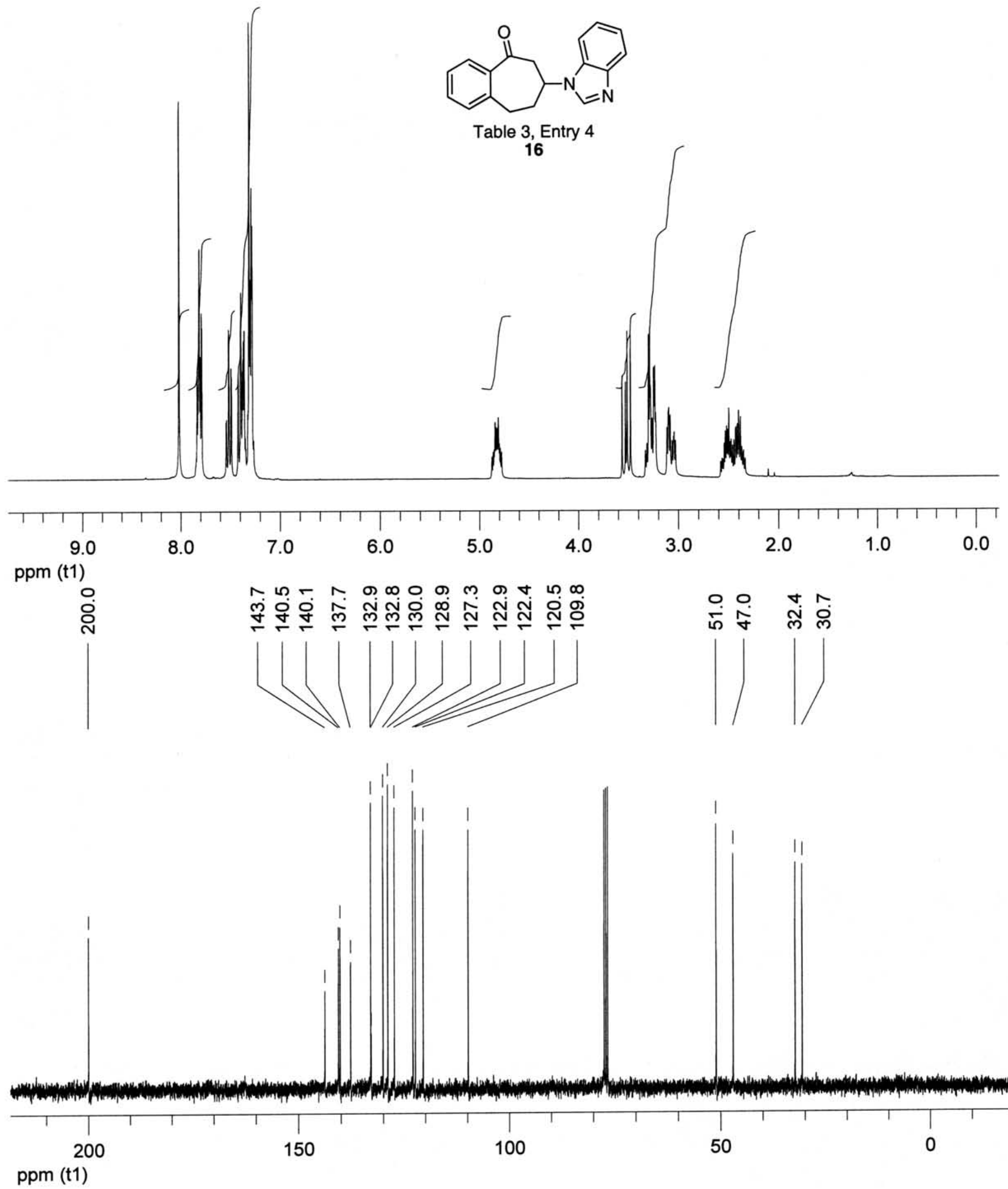




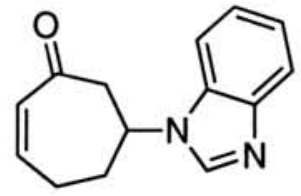

Table 3, Entry 5

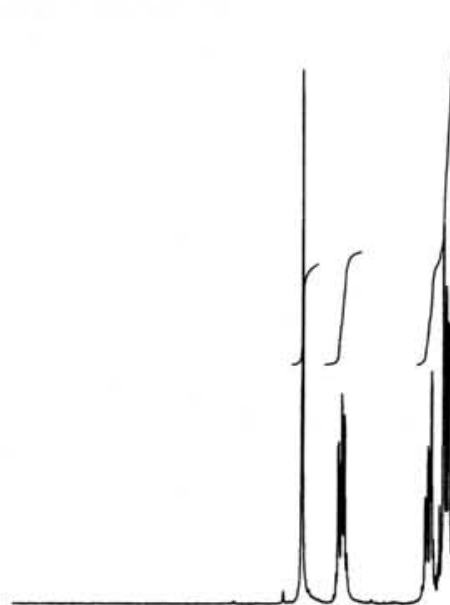
18

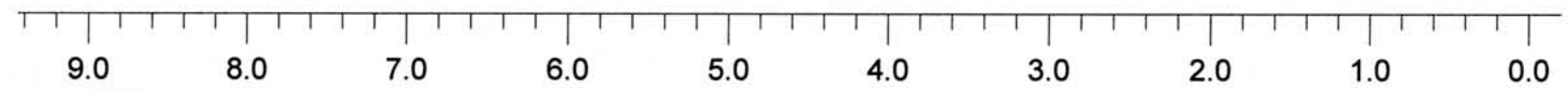
ppm (t1)

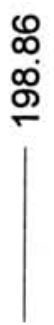

음유

过 过

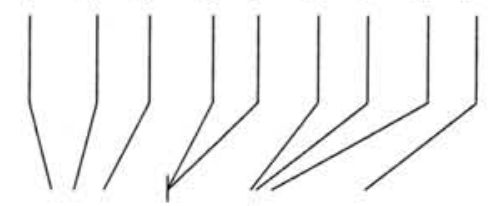

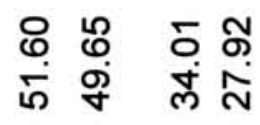
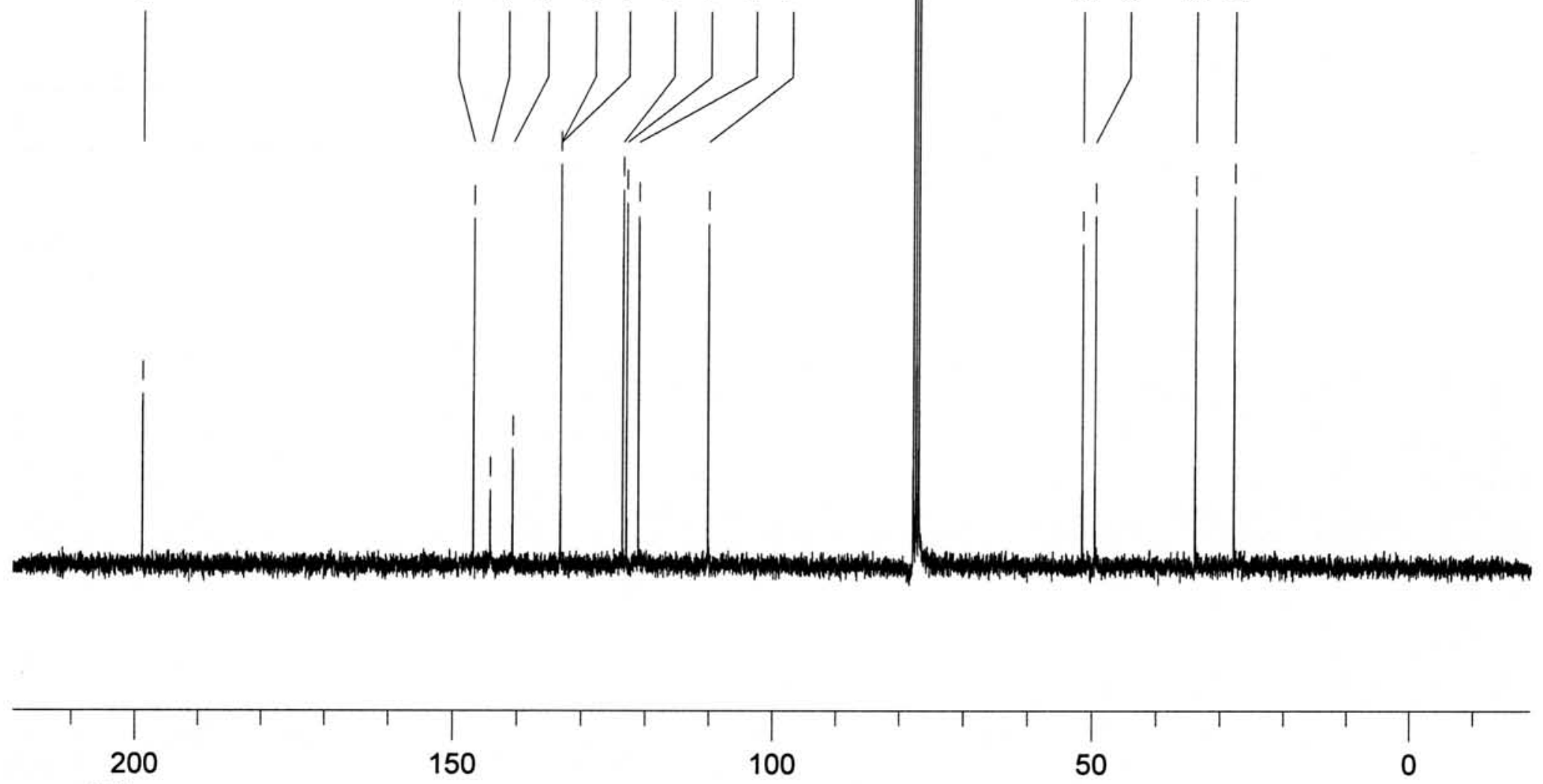

ppm (t1) 


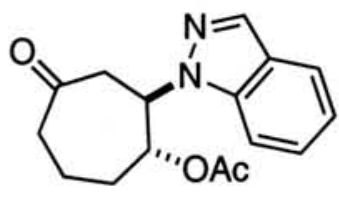

Table 3, Entry 6 20
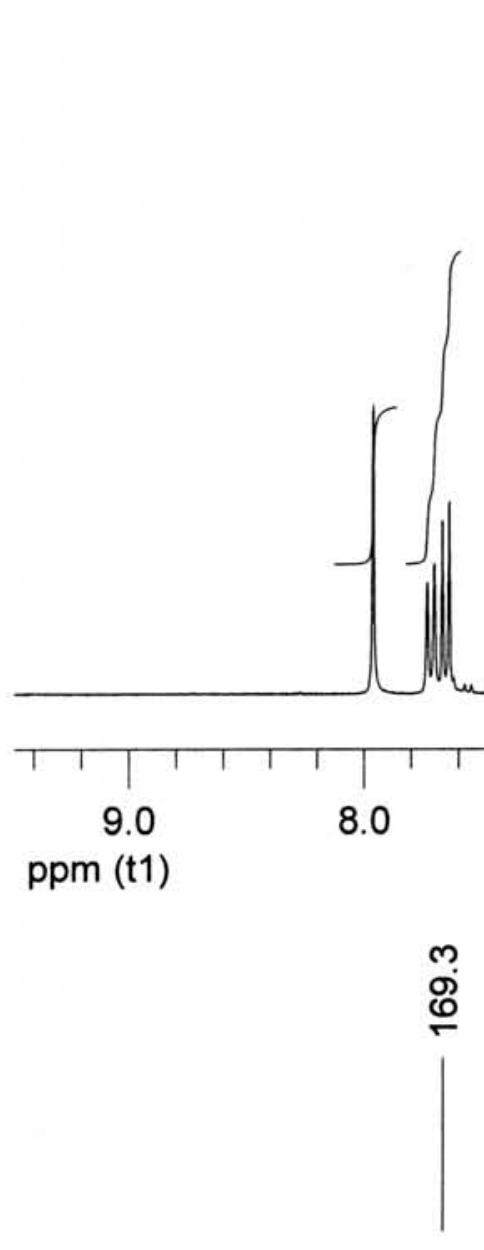

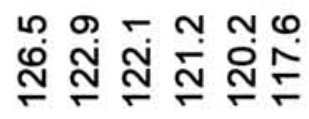
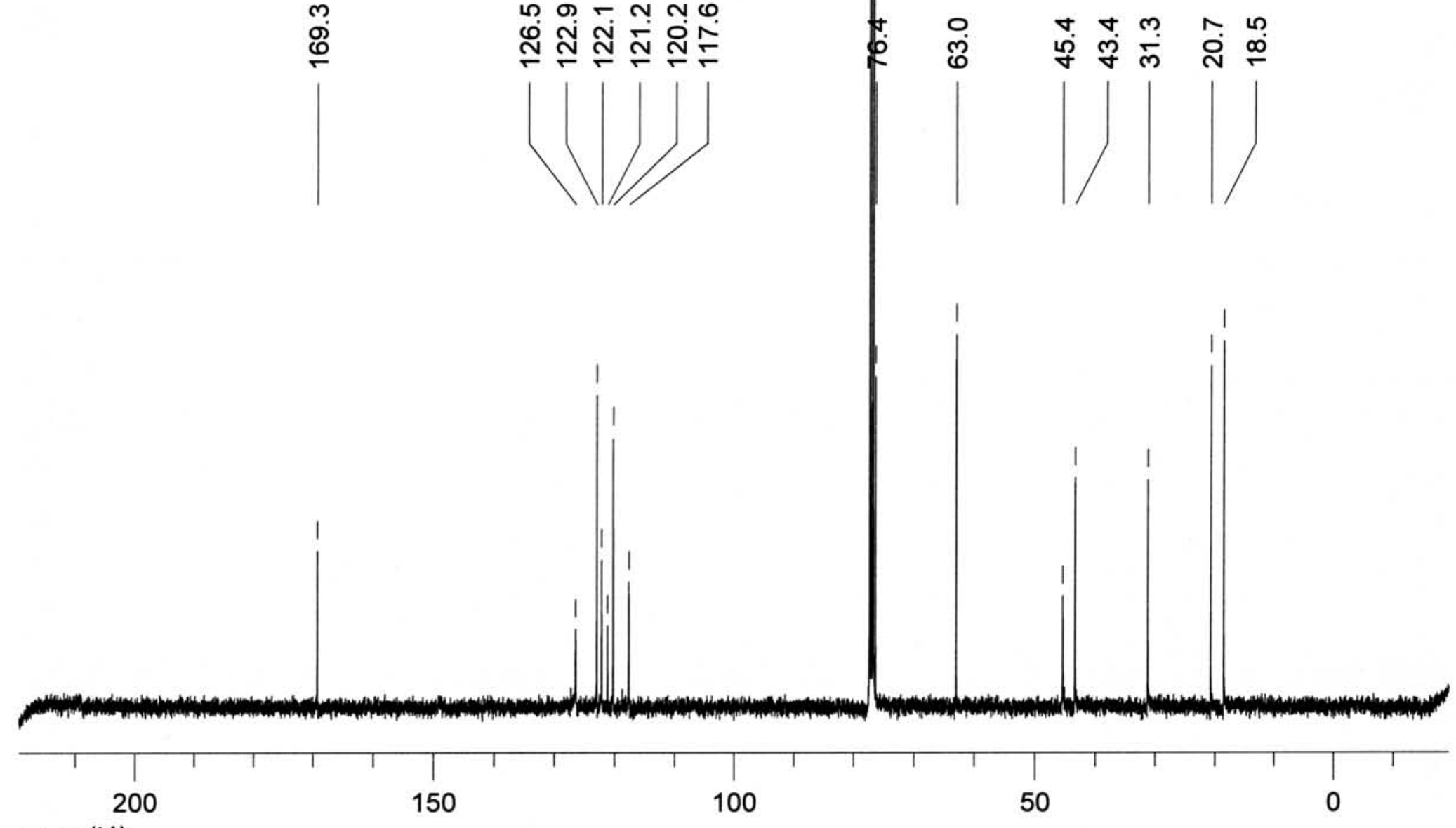

ppm (t1) 


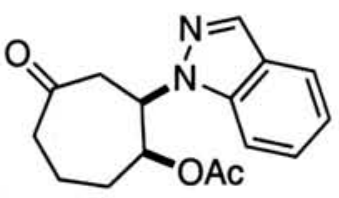

Table 3, Entry 6 20
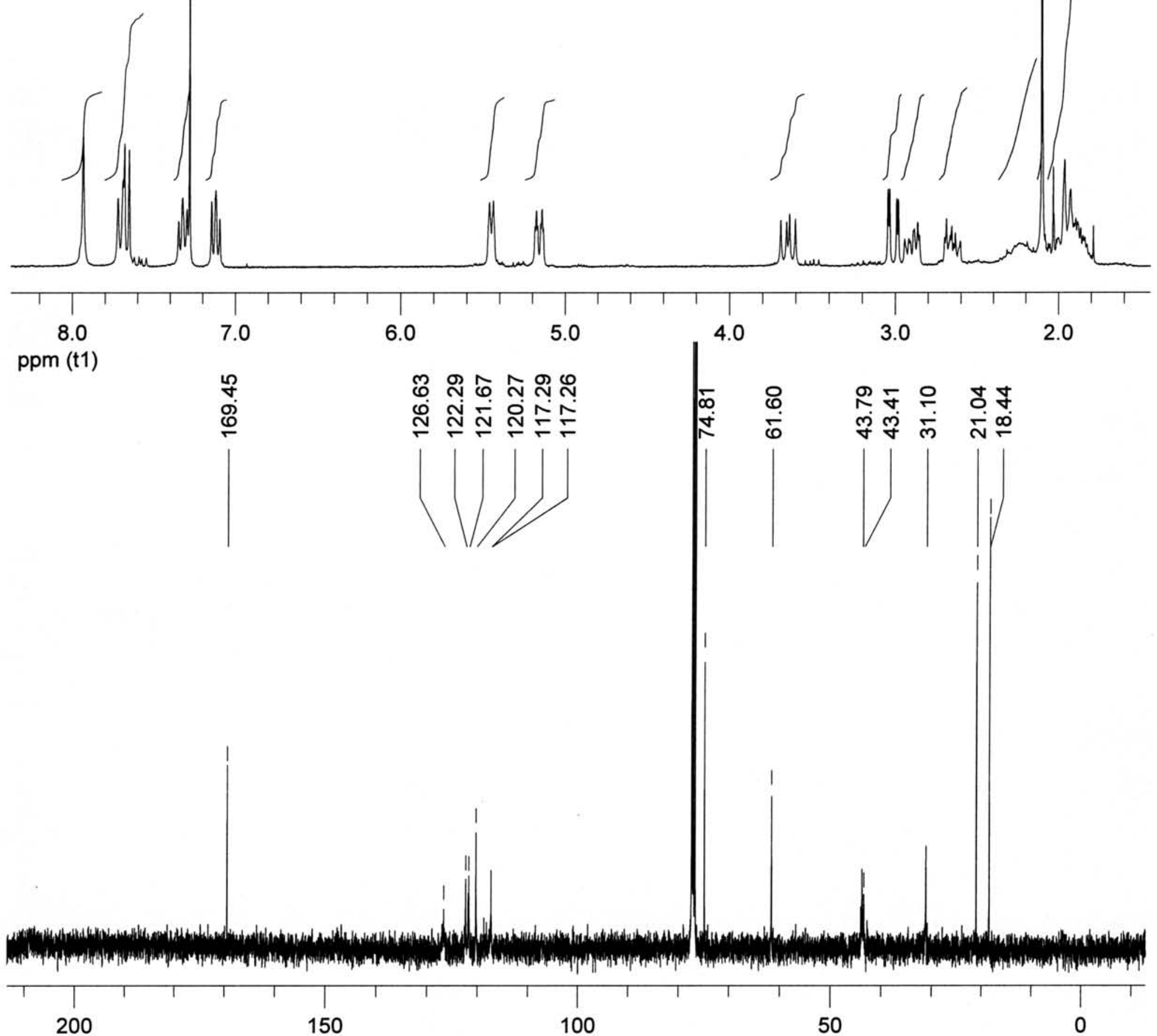

ppm (t1) 


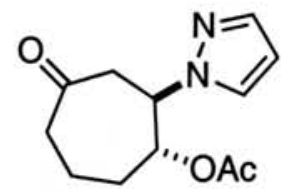

Table 3, Entry 7

21

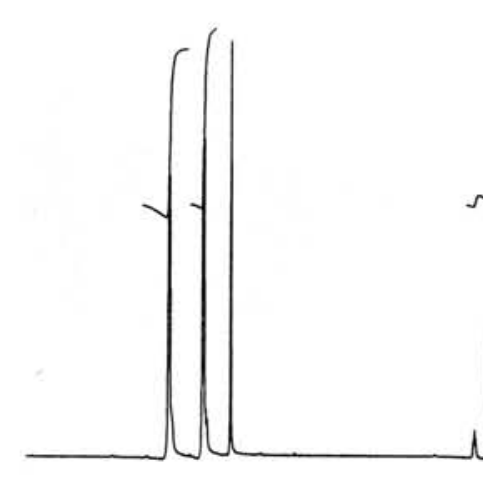

1
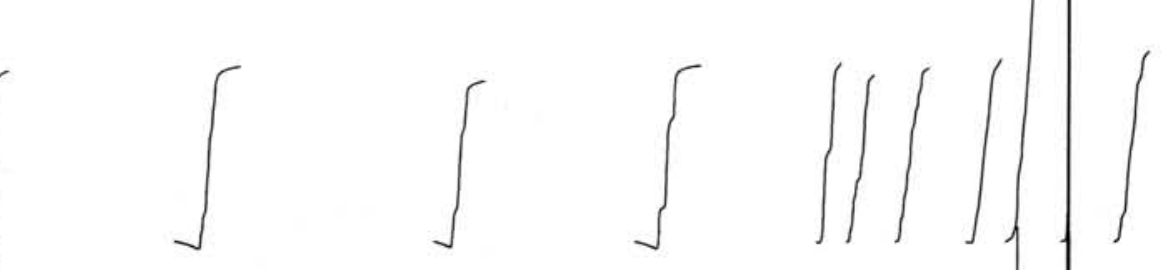

$$
\text { T }
$$




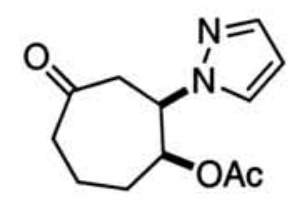

Table 3, Entry 7 21
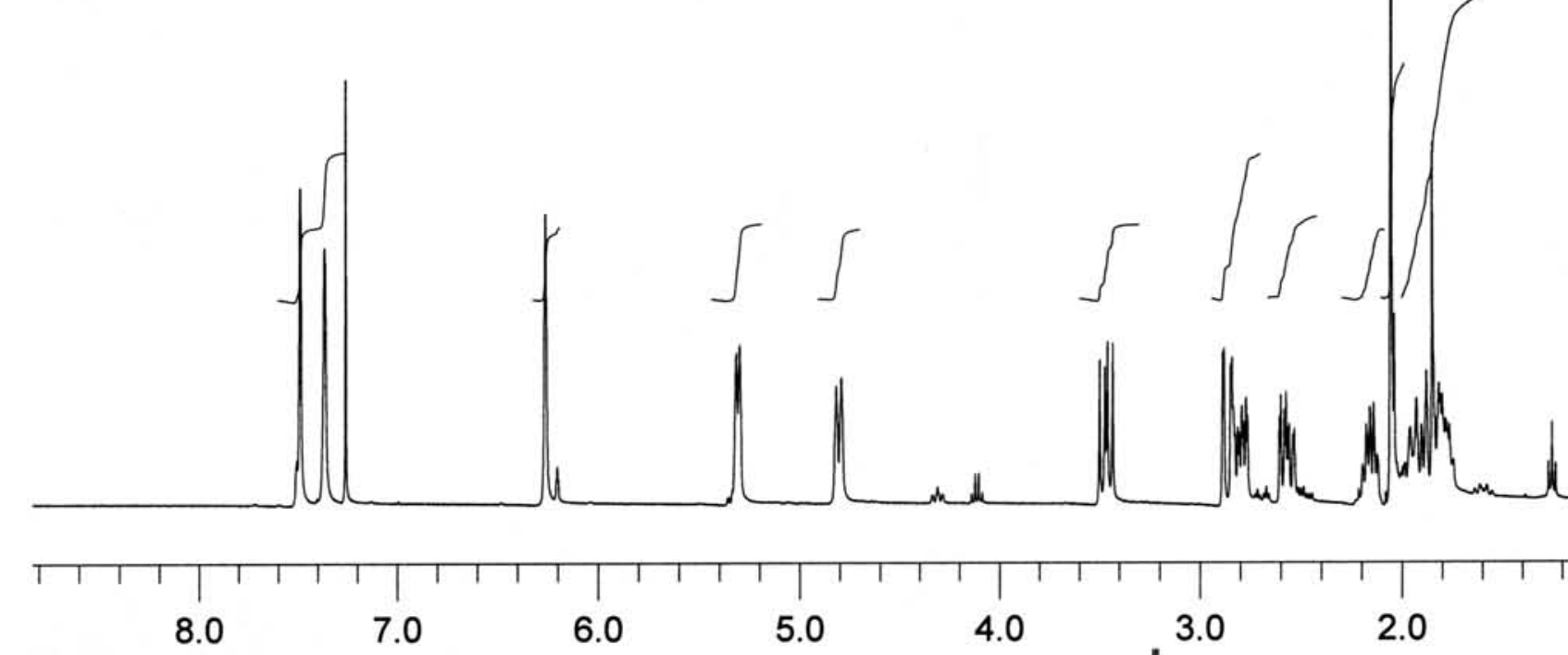
ppm (t1)
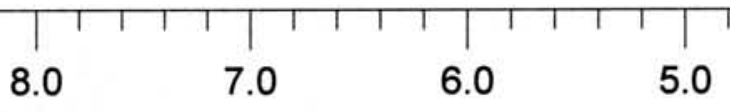

5.0

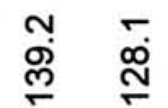

$\check{\phi}$

4.0

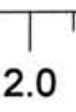

3.0

2.0

1.0

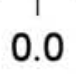

箇

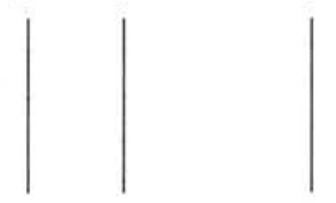

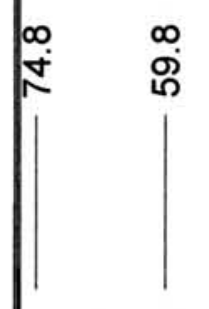
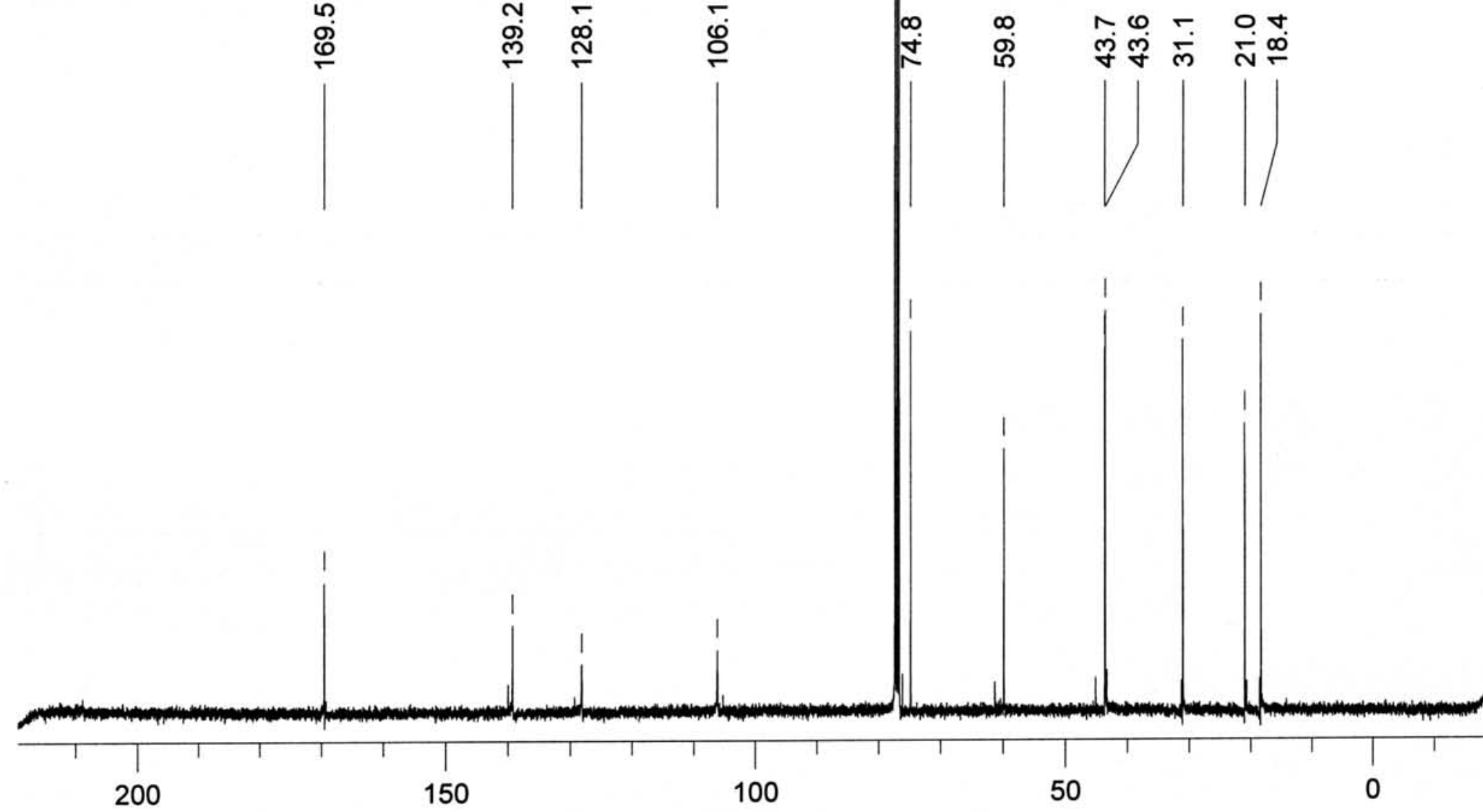

ppm (t1) 
<smiles>[2H]c1nc2ccccc2[nH]1</smiles>

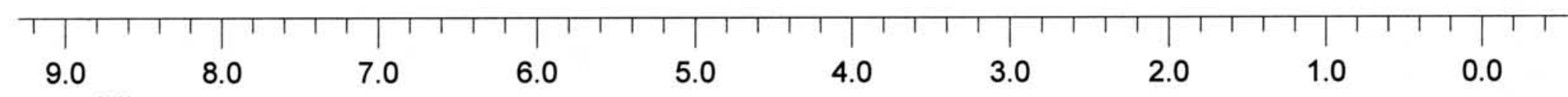
ppm (t1)

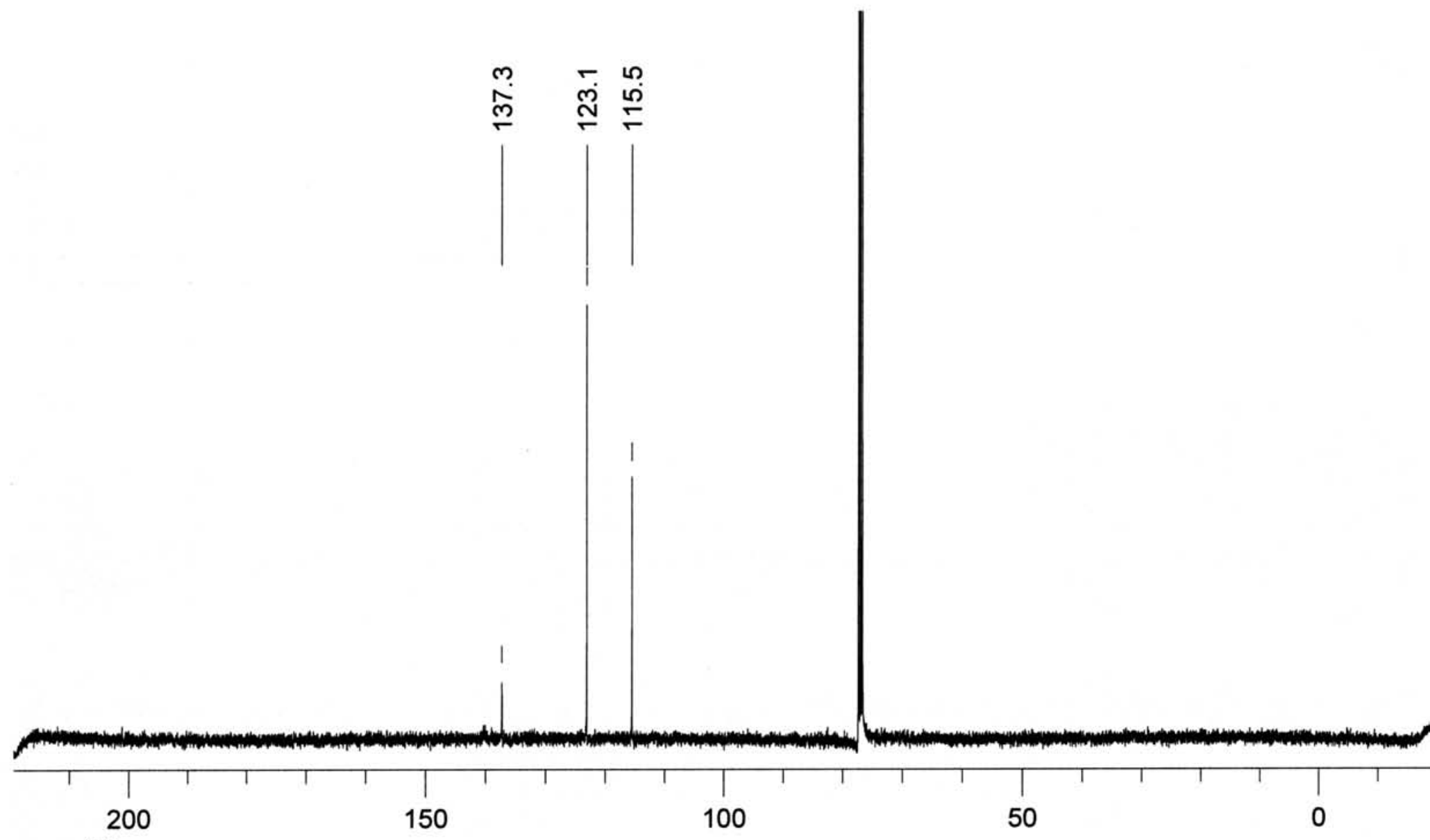

ppm (t1) 
<smiles>[2H]c1nc2ccccc2n1[C@@H]1CCCCC(=O)[C@H]1[2H]</smiles>

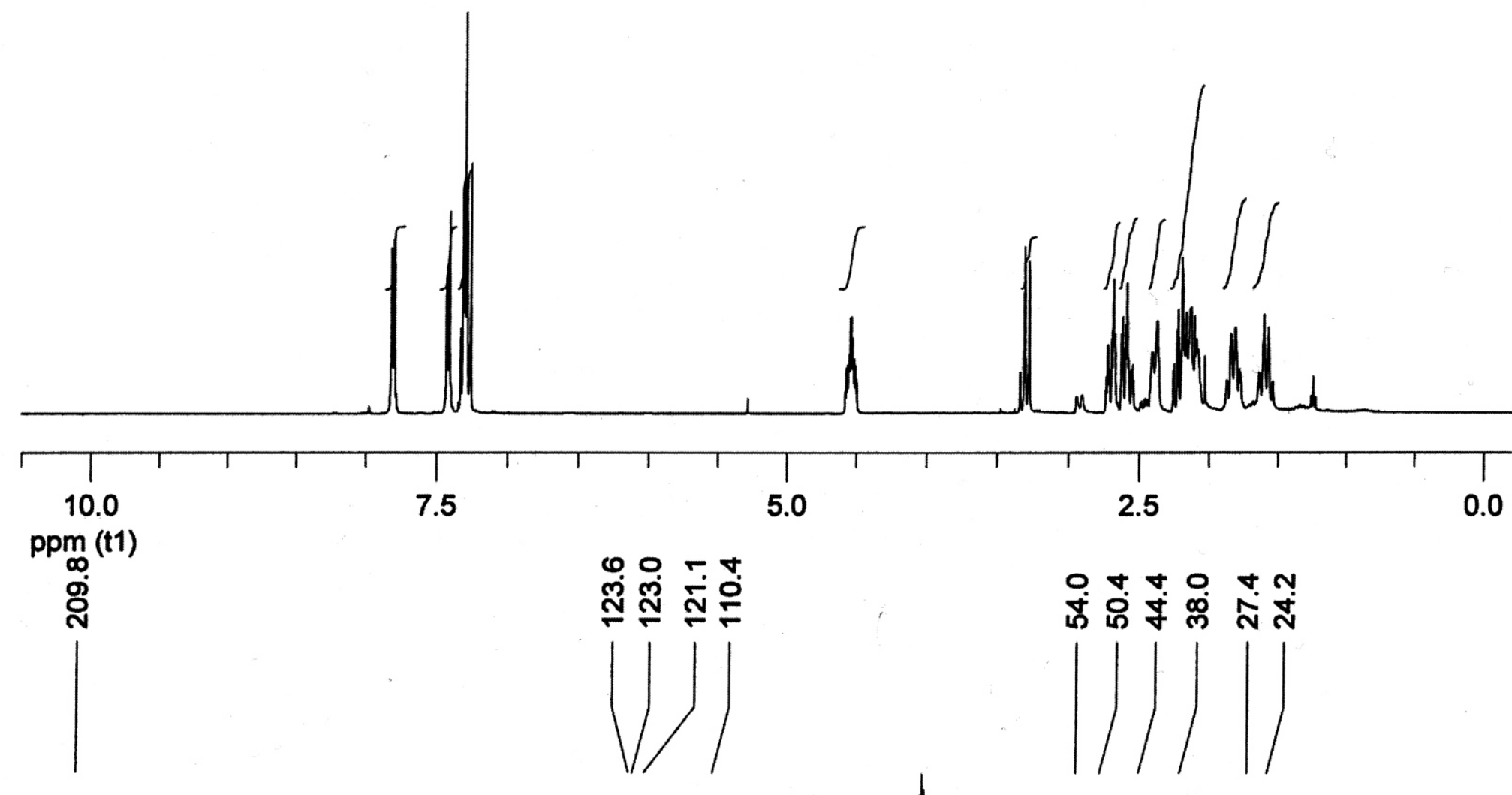

